Keywords:

DWPF

Canister

Thermal

Retention: permanent

\title{
COMSOL Multiphysics Model for DWPF Canister Filling
}

M.R.Kesterson

March 31, 2011

Applied Computational Engineering and Statistics Savannah River National Laboratory

Aiken, SC 29808

This document was prepared in conjunction with work accomplished under Contract No. DE-AC09-08SR22470 with the U.S. Department of Energy.

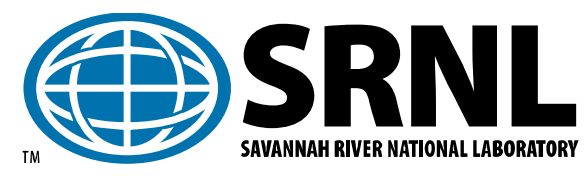




\section{DISCLAIMER}

This work was prepared under an agreement with and funded by the U.S. Government. Neither the U.S. Government or its employees, nor any of its contractors, subcontractors or their employees, makes any express or implied: 1 . warranty or assumes any legal liability for the accuracy, completeness, or for the use or results of such use of any information, product, or process disclosed; or 2. representation that such use or results of such use would not infringe privately owned rights; or 3. endorsement or recommendation of any specifically identified commercial product, process, or service. Any views and opinions of authors expressed in this work do not necessarily state or reflect those of the United States Government, or its contractors, or subcontractors.

This document was prepared in conjunction with work accomplished under Contract No. DE-AC09-08SR22470 with the U.S. Department of Energy. 


\section{AUTHORS:}

M.R, Kesterson, Applied Computational Engineering and Statistics

Date Savannah River National Laboratory

\section{TECHNICAL REVIEWERS:}

N.K.Gupta, Applied Computational Engineering and Statistics

Date Savannah River National Laboratory

\section{APPROVERS:}

J.W. Amoroso, Customer

Date

Process Technology Programs

A.L. Billings, Customer

Date

Process Technology Programs

P.L. Lee, Manager Applied Computational Engineering and Statistics

Date

Savannah River National Laboratory 


\section{Table of Contents}

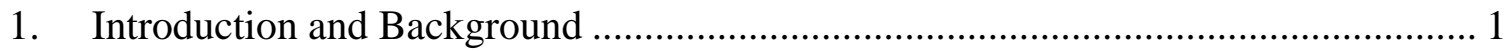

2. Model Description …………………………............................................... 1

3. Inputs and Assumptions ................................................................................. 3

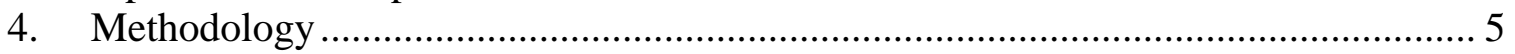

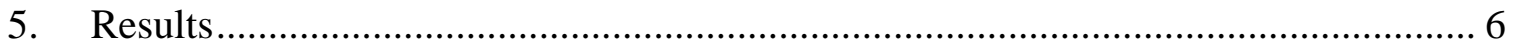

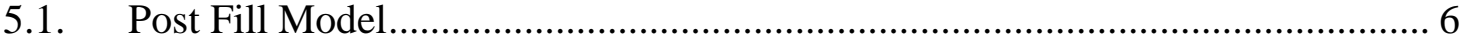

5.2. Glass Pouring Model................................................................................. 7

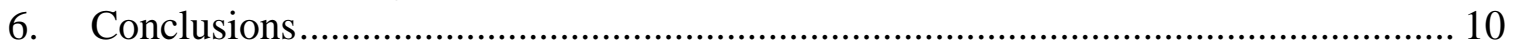

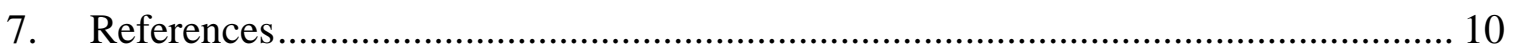

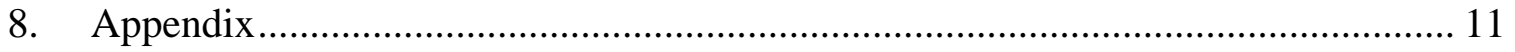

Appendix A - Comsol Constants and Expressions ......................................................... 11

Appendix B - Simulation Temperature versus Time Data........................................... 14

\section{List of Tables}

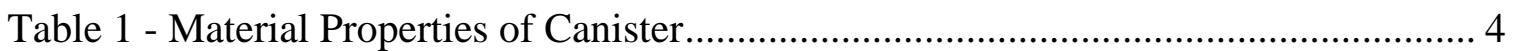

Table 2 - Material Properties of DWPF glass ............................................................. 4

\section{List of Figures}

Figure 1 - COMSOL Multiphysics model of the DWPF canister. ……………………...... 2

Figure 3 - Temperature profile for 15 inch thermocouple during the cool down phase..... 6

Figure 4 - Temperature profile for 51 inch thermocouple during the cool down phase..... 7

Figure 5 - Temperature curves for thermocouple and simulation data located on the outside bottom of the canister.................................................................. 8

Figure 6 - Temperature curves for thermocouple and simulation data at the 15 inch location of the canister................................................................................. 8

Figure 7 - Temperature curves for thermocouple and simulation data at the 51 inch location of the canister......................................................................... 9

Figure 8 - Temperature curves for thermocouple and simulation data at the 87 inch location of the canister................................................................................. 9

Figure 9 - Temperature curves for thermocouple and simulation data at the 99 inch location of the canister. 


\section{Introduction and Background}

The purpose of this work was to develop a model that can be used to predict temperatures of the glass in the Defense Waste Processing Facility (DWPF) canisters during filling and cooldown. Past attempts to model these processes resulted in large $(>200 \mathrm{~K})$ differences in predicted temperatures compared to experimentally measured temperatures. This work was therefore intended to also generate a model capable of reproducing the experimentally measured trends of the glass/canister temperature during filling and subsequent cooldown of DWPF canisters.

To accomplish this, a simplified model was created using the finite element modeling software COMSOL Multiphysics [1] which accepts user defined constants or expressions to describe material properties. The model results were compared to existing experimental data [2] for validation.

This work was funded by the Department of Energy (DOE) Office of Environmental Management (EM) technology development task EM-31 WP-5.1.2.

\section{Model Description}

A simplified model for the DWPF canister and interior space consisting of 13,712 elements was created in Comsol Multiphysics and is shown in Figure 1. The model did not incorporate convective heat transfer ordinarily modeled using Navier-Stokes equations for fluid flow. Rather, a model was developed that incorporated heat transfer by conduction, with the radiation and convection terms approximated by a generalized heat loss function during the canister filling cycle. The reason for developing this type of model was two-fold; 1) the model used a relatively long duration (3000 minutes) timeframe that coupled with the complex nature of the Navier-Stokes equations would extend the computation times to impractical limits and, 2) the convective heat transfer occurring in the glass during pouring was considered minimal below the glass transition temperature. 


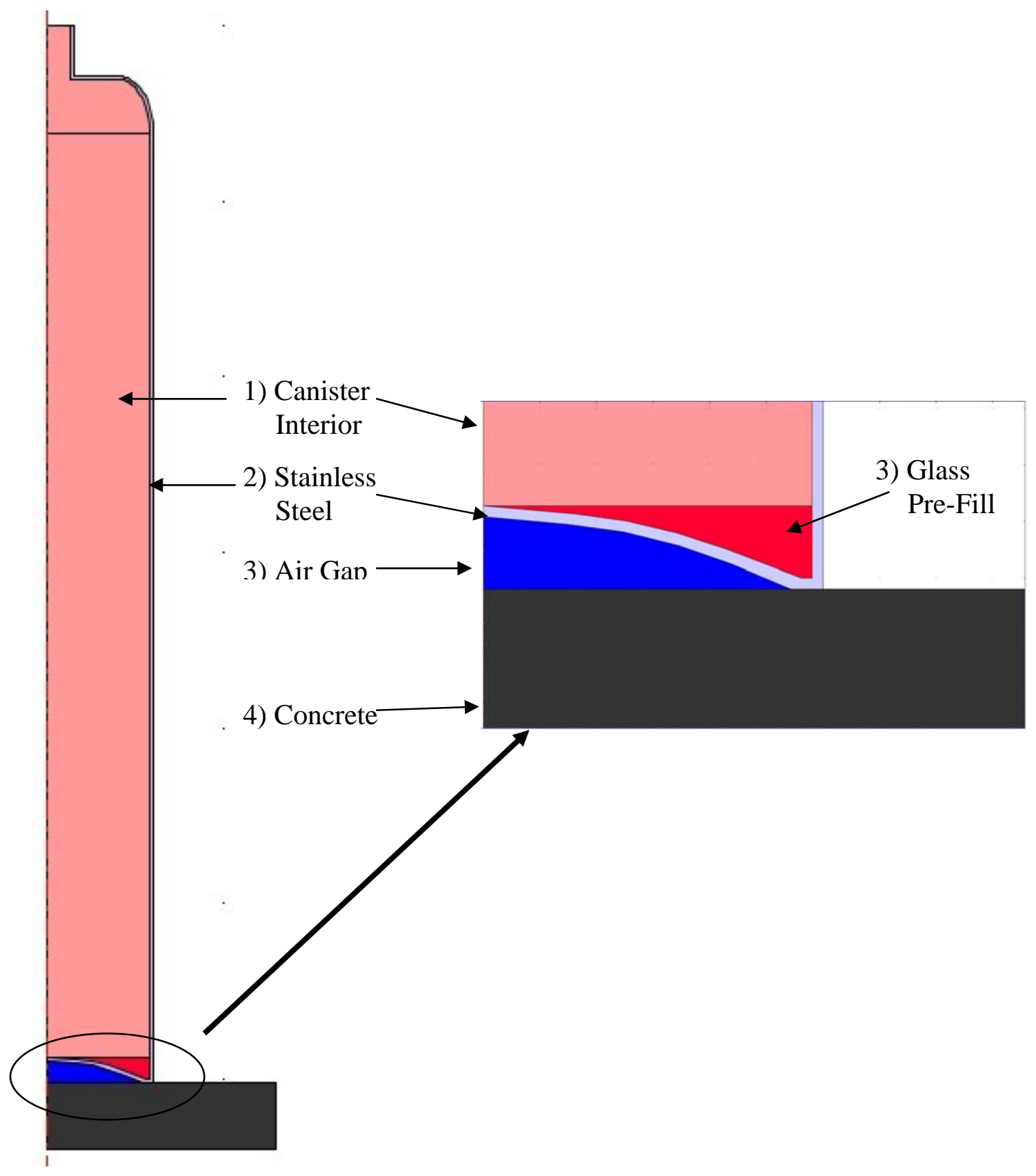

Figure 1 - COMSOL Multiphysics model of the DWPF canister.

For initial stabilization of the simulations, the model contained a small region pre-filled with glass with a volume equivalent to the first 10 minutes of pouring. The region is initialized to the thermal properties of the glass with an initial temperature of $200^{\circ} \mathrm{C}$. This region added a buffer to the sudden transition from air to glass and the resultant change in thermal mass within the canister.

The model incorporated convective heat transfer on the outer surface of the canister as well as radiative losses to ambient. Internally, the walls of the canister exchanged with the glass via radiative heat transfer from the surface of the glass. 
In order to model convection and radiative losses from the glass surface, a parameter called Q_loss was employed. Q_loss was designed to encompass to cumulative effect of the radiation losses at the surface along with convection losses below the top surface of the glass:

$$
\text { Q_loss }=Q_{\text {radiation }}+Q_{\text {convection }}
$$

The Q_loss parameter employed in the model was a function of the fluid height and varied with radial position:

$$
\text { Q_loss }=\text { Q_loss_base* }(\mathrm{f}(\mathrm{z}) * \mathrm{f}(\mathrm{r}))
$$

where Q_loss_base is a constant. In order to obtain these functions based on height and radial distance, the temperature as a function of height was plotted for the initial cooldown period for each thermocouple location. A general quadratic term was fit to the data and written into the COMSOL model. The model was run and the coefficients to the fit were modified until a good fit between the simulation output and the experimental data was achieved. For the Q_loss function, it was found that a quadratic equation coupled with an exponential decrease based on radial position, reasonably approximated the experimental data. The final form of the equation is shown in Appendix A - Global Expressions table.

\section{Inputs and Assumptions}

Below are the inputs and assumptions that are used in the COMSOL model. All units reported below are used in the model. The COMSOL model has a base unit system of SI, but the user is allowed to input properties in any units and COMSOL converts them to the base unit system for calculations.

Assumptions:

3.1. The ambient temperature in the room is a constant $26^{\circ} \mathrm{C}$. The experimental data shows that the room temperature had variations between $24^{\circ} \mathrm{C}$ and $30^{\circ} \mathrm{C}$ [2] with an average room temperature that was approximately $26^{\circ} \mathrm{C}$.

3.2. Radiation and convective heat transfer in the enclosed space between the canister bottom and the ground is accounted for by an increased thermal conductivity term.

3.3. The experimental data used was based on scale glass melter run with a nominal pour rate of $228 \mathrm{lb} / \mathrm{hr}$ [2]. In reviewing the experimental data, the actual flow rate varied between 190 and $360 \mathrm{lb} / \mathrm{hr}$. A step function was employed in the model to approximate the flow rate variation and more accurately match the experimental conditions. The function is shown in Appendix A - Global Expressions table 
Inputs:

3.4. Glass pour temperature is $1080^{\circ} \mathrm{C}$

3.5. Canister dimensions are supplied on drawing W747391 [3]

3.6. Material Properties - Table 1 and Table 2

Table 1 - Material Properties of Canister

\begin{tabular}{|c|c|c|c|c|c|}
\hline Material & \multicolumn{2}{|c|}{ Thermal Conductivity } & Density & \multicolumn{2}{|c|}{ Specific Heat } \\
\hline \multirow{8}{*}{$\begin{array}{l}\text { Stainless } \\
\text { Steel } \\
\text { Canister } \\
{[4]}\end{array}$} & $\frac{B T U}{h r \cdot l b_{m} \cdot{ }^{o} F}$ & Temperature $\left({ }^{\circ} \mathrm{F}\right)$ & \multirow[t]{8}{*}{494.429} & \multirow[t]{2}{*}{$\frac{B T U}{l b_{m} \cdot{ }^{o} F}$} & \multirow[t]{2}{*}{$\begin{array}{l}\text { Temperature } \\
\text { (F) }\end{array}$} \\
\hline & 3.9915 & -328 & & & \\
\hline & 6.28963 & -148 & & \multirow[t]{2}{*}{0.120} & \multirow[t]{2}{*}{32} \\
\hline & 7.74108 & 32 & & & \\
\hline & 9.43444 & 212 & & \multirow[t]{2}{*}{0.135} & \multirow[t]{2}{*}{752} \\
\hline & 12.5793 & 932 & & & \\
\hline & 14.9983 & 1292 & & \multirow[t]{2}{*}{0.135} & \multirow[t]{2}{*}{3000} \\
\hline & 14.9983 & 3000 & & & \\
\hline
\end{tabular}

Table 2 - Material Properties of DWPF glass

\begin{tabular}{|c|c|c|c|c|c|c|}
\hline \multirow{2}{*}{$\begin{array}{l}\text { Material } \\
\text { Glass } \\
\text { [4] }\end{array}$} & \multicolumn{2}{|c|}{ Thermal Conductivity } & \multicolumn{2}{|c|}{ Density } & \multicolumn{2}{|c|}{ Specific Heat } \\
\hline & \multirow[t]{2}{*}{$\frac{B T U}{h r \cdot l b_{m} \cdot{ }^{o} F}$} & \multirow[t]{2}{*}{$\begin{array}{c}\text { Temperature } \\
\left({ }^{\circ} \mathrm{F}\right)\end{array}$} & \multirow[t]{2}{*}{$\left(\mathrm{lb} / \mathrm{ft}^{3}\right)$} & \multirow[t]{2}{*}{$\begin{array}{l}\text { Temperature } \\
\left({ }^{\circ} \mathrm{F}\right)\end{array}$} & $\frac{B T U}{l b_{m} \cdot{ }^{o} F}$ & $\begin{array}{l}\text { Temperature } \\
\left({ }^{\circ} \mathrm{F}\right)\end{array}$ \\
\hline & & & & & 0.204 & 20 \\
\hline & \multirow[b]{2}{*}{0.76449} & \multirow[b]{2}{*}{20} & \multirow[t]{2}{*}{172.31} & \multirow[t]{2}{*}{20} & 0.204 & 68 \\
\hline & & & & & 0.24 & 212 \\
\hline & \multirow[b]{2}{*}{0.76449} & \multirow[b]{2}{*}{68} & \multirow[t]{2}{*}{171.37} & \multirow[t]{2}{*}{392} & 0.272 & 392 \\
\hline & & & & & 0.295 & 572 \\
\hline & \multirow[b]{2}{*}{0.76449} & \multirow[b]{2}{*}{1742} & \multirow[t]{2}{*}{167.31} & \multirow{2}{*}{932} & 0.312 & 752 \\
\hline & & & & & 0.325 & 932 \\
\hline & \multirow[b]{2}{*}{1.3741} & \multirow[b]{2}{*}{1832} & \multirow{2}{*}{165.44} & \multirow[t]{2}{*}{112} & 0.334 & 1112 \\
\hline & & & & & 0.342 & 1292 \\
\hline & \multirow[b]{2}{*}{2.8063} & \multirow[b]{2}{*}{2012} & \multirow[t]{2}{*}{162.94} & \multirow[t]{2}{*}{1292} & 0.349 & 1472 \\
\hline & & & & & 0.354 & 1652 \\
\hline & \multirow[b]{2}{*}{3.5079} & \multirow[b]{2}{*}{2102} & \multirow[t]{2}{*}{160.45} & \multirow[t]{2}{*}{1472} & 0.356 & 1740 \\
\hline & & & & & 0.358 & 1832 \\
\hline & \multirow{4}{*}{3.5079} & \multirow{4}{*}{3000} & \multirow[t]{2}{*}{157.95} & \multirow[t]{2}{*}{1652} & 0.362 & 2012 \\
\hline & & & & & 0.363 & 2102 \\
\hline & & & 151.77 & 2192 & 0.365 & 2192 \\
\hline & & & & & 0.365 & 3000 \\
\hline
\end{tabular}

The model consisted of two additional regions requiring material properties. These regions were the floor below the canister and the air initially contained within the canister and the air trapped between the canister and the concrete floor. The floor was modeled as 
a concrete slab with a lower temperature boundary condition equal to the ambient air temperature. The material library contained within COMSOL is used to obtain the material properties for both the air and concrete.

\section{Methodology}

Two types of simulations were conducted 1) a post fill simulation and 2) a full simulation that incorporated both filling and cooldown. The purpose for the post fill simulation was to obtain values for the external heat transfer coefficients, particularly due to radiation effects. For this simulation, the model was initialized with a temperature distribution seen in the experimental data at a time of 1074 minutes, a time that is sufficiently past the end of pour time such that heat/glass addition and flow effects can be ignored. Using this temperature distribution as the starting point, the simulation was run to the final 3000 minutes. In this timeframe, the heat loss from the system is due to convective flow at the surface of the canister and radiative effects. The convection heat transfer at the surface of the canister is modeled by generalized equations based on air properties and temperature of the canister surface and the ambient temperature. In addition to the convection heat transfer, radiative heat transfer occurs from the canister surface. The post fill model holds the view factor for the radiation terms constant and the emissivity value was varied in order to match the simulation output with the experimental data.

Once the external heat transfer terms were refined in the post fill case, the parameters were applied to the full model that incorporates both the filling and cooldown phase of the experiment. The full simulation was run in discrete timesteps, typically 40 second increments. A larger time step, up to 240 seconds, was used for rough estimates yielding temperature deviations of approximately $5 \%$ over the smaller timestep. Using a timestep of 80 seconds, the temperature deviation was approximately 1\%.

Based on the time of the simulation and the time step, a corresponding volume of glass was added to the system by changing the material properties of the subdomain in the region between the calculated new glass height and the previous glass height (glass_height(t) - glass_height(dt)). The material properties for this region were changed from air to those of the glass, specifically changing the specific heat, density, and thermal conductivity. In making this change, heat was also added to the region, since temperature could not be directly imposed. The amount of heat added to the system was based on the specific heat, volume, and density of the differential volume of glass and the difference between the region's current temperature and the temperature of the glass melt.

The temperature of the glass melt was fit to experimental data. The maximum temperatures reported by the thermocouples were plotted as a function of thermocouple location. The curve fit for this data was used in the COMSOL model to define the temperature of the top surface of the glass as it rises in the canister during glass pouring.

In addition to the heat being added to bring the glass up to the correct temperature, a Q_loss term was used to remove heat from the system. This heat loss removal is dependent on the glass height, and radial position in the canister. The actual form of the equation used for Q_loss is shown in Appendix A - Global Expressions table. The 
parameters, namely Q_loss_base and how much heat is removed based on radial position and height were obtained based on trial and error fits to the experimental data.

\section{Results}

\subsection{Post Fill Model}

Simulations were run in which the canister was pre-filled with glass to a level of $2.38 \mathrm{~m}$, and a temperature distribution in the glass was set to be equal to that seen in the experimental data for 1074 minutes (approximately 150 minutes after the end of the glass pour cycle). The 1074 minutes temperature profile was chosen as the starting point for the cooldown of the glass without additional heat input during the pour phase. The simulation was then run to the full 3000 minutes corresponding to the last of the experimental data and the results were compared to the experimental values. The emissivity value for radiative heat transfer was modified to achieve good agreement with the experimental data as shown in Figure 2 and Figure 3. The results were obtained using a surface emissivity of 0.8 and an external view factor of 1.0.

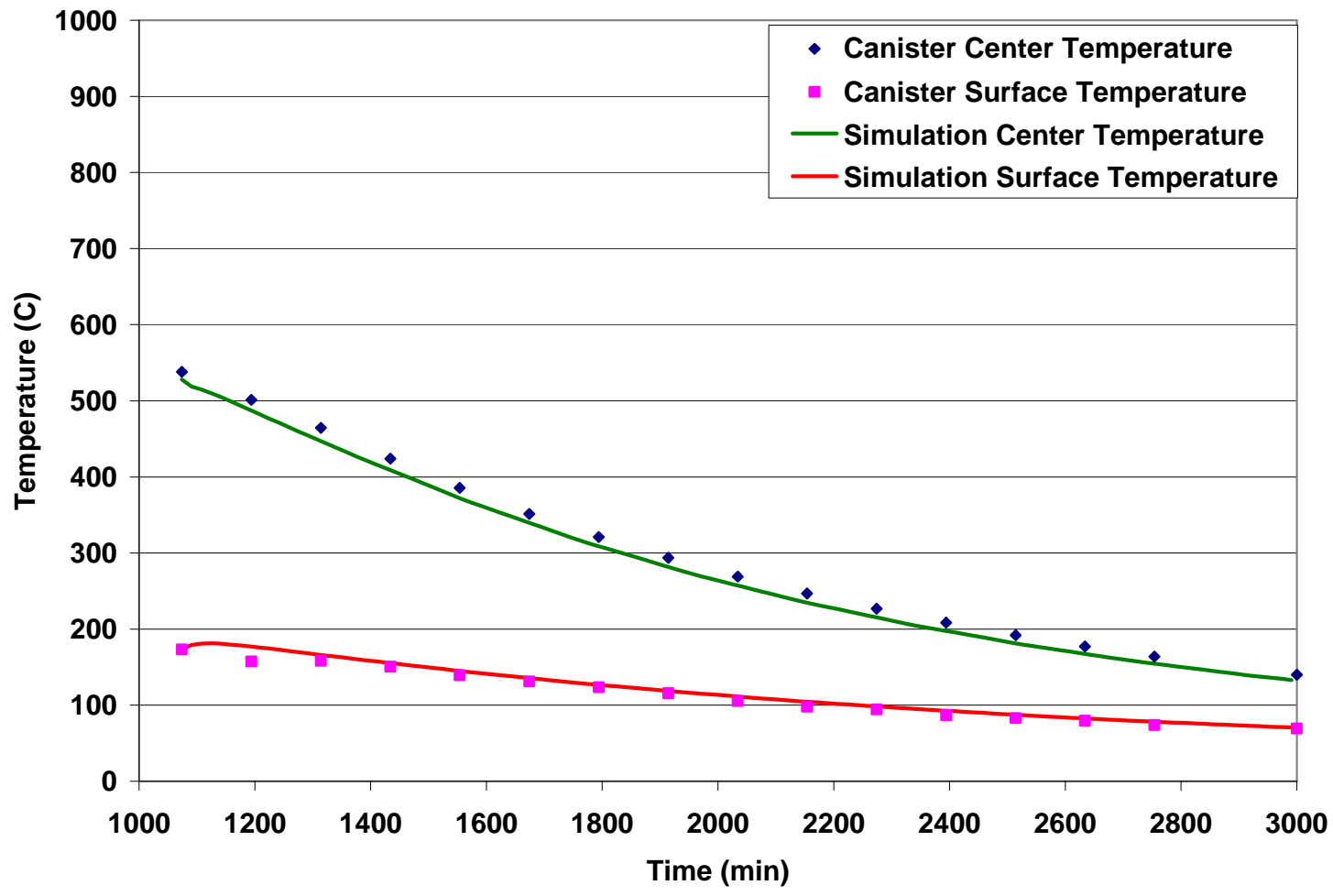

Figure 2 - Temperature profile for 15 inch thermocouple location during the cool down phase. 


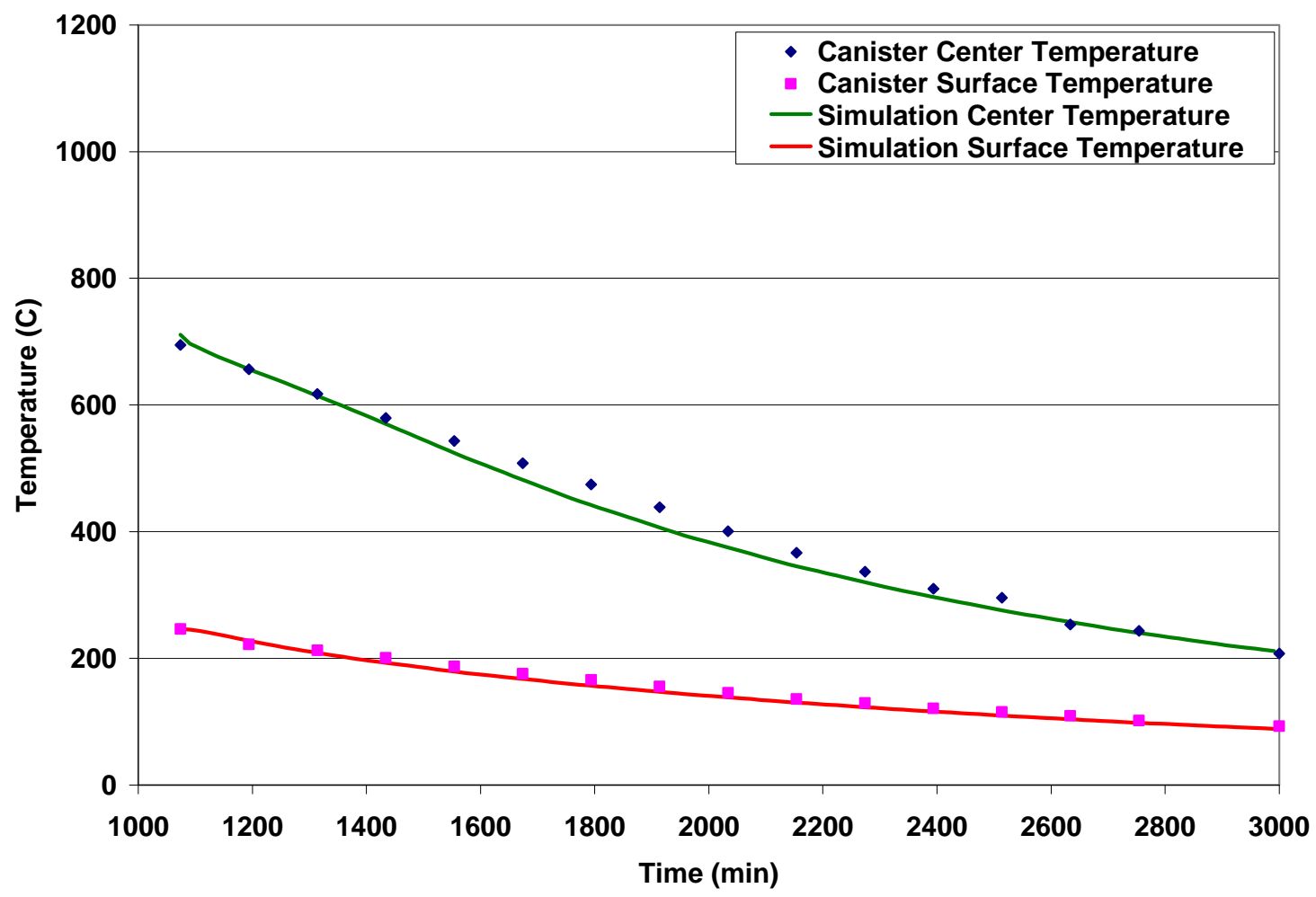

Figure 3 - Temperature profile for 51 inch thermocouple location during the cool down phase.

\subsection{Glass Pouring Model}

Once the parameters for radiative heat loss for the outside surface of the container were verified, the model was run through an iterative filling process in which the Q_loss term and internal surface radiation terms were varied in order to approximate the temperature profile seen in the experimental data [2].

Figures Figure 4 to Figure 8 show the final results obtained from the model at five thermocouple locations: 1) bottom surface of the canister, 2) 15 inches, 3) 51 inches, 4) 87 inches, and 5) 99 inches. The larger temperature differences predicted at the 99 inch height are due to the lack of internal radiation once the pour cycle is complete. During the pour phase the internal radiation loss was based on the canister internal surface temperature and the calculated glass temperature.

As seen in the following figures, the temperatures of the glass and the canister surface predicted using the model compare relatively well with the experimental data for both filling and cooling. For the end time of 3000 minutes, the simulations predict a temperature between 30 and $45{ }^{\circ} \mathrm{C}$ (at 15 and 87 inches respectively) higher than the experimental data. 


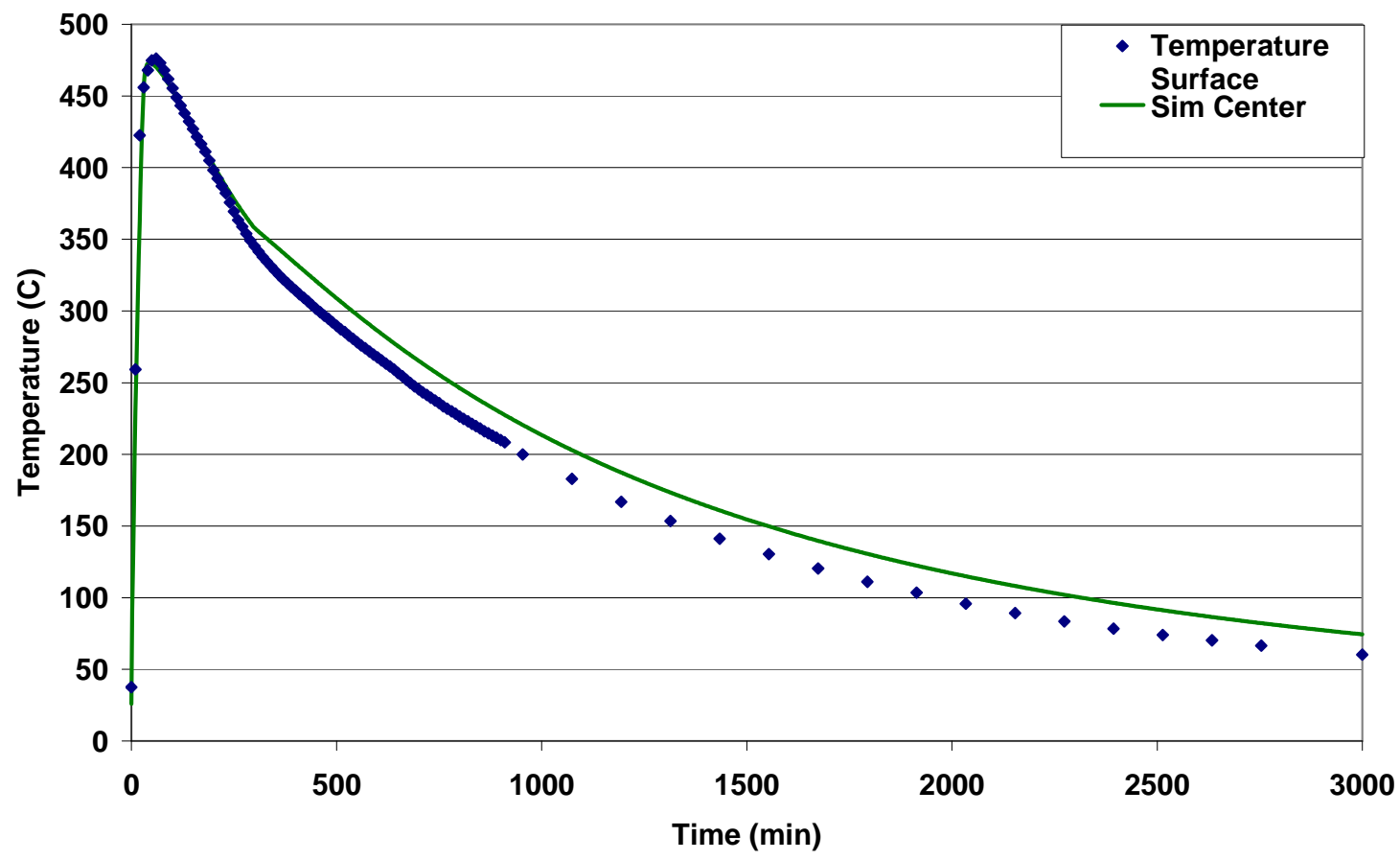

Figure 4 - Temperature curves for thermocouple and simulation data located on the outside bottom of the canister.

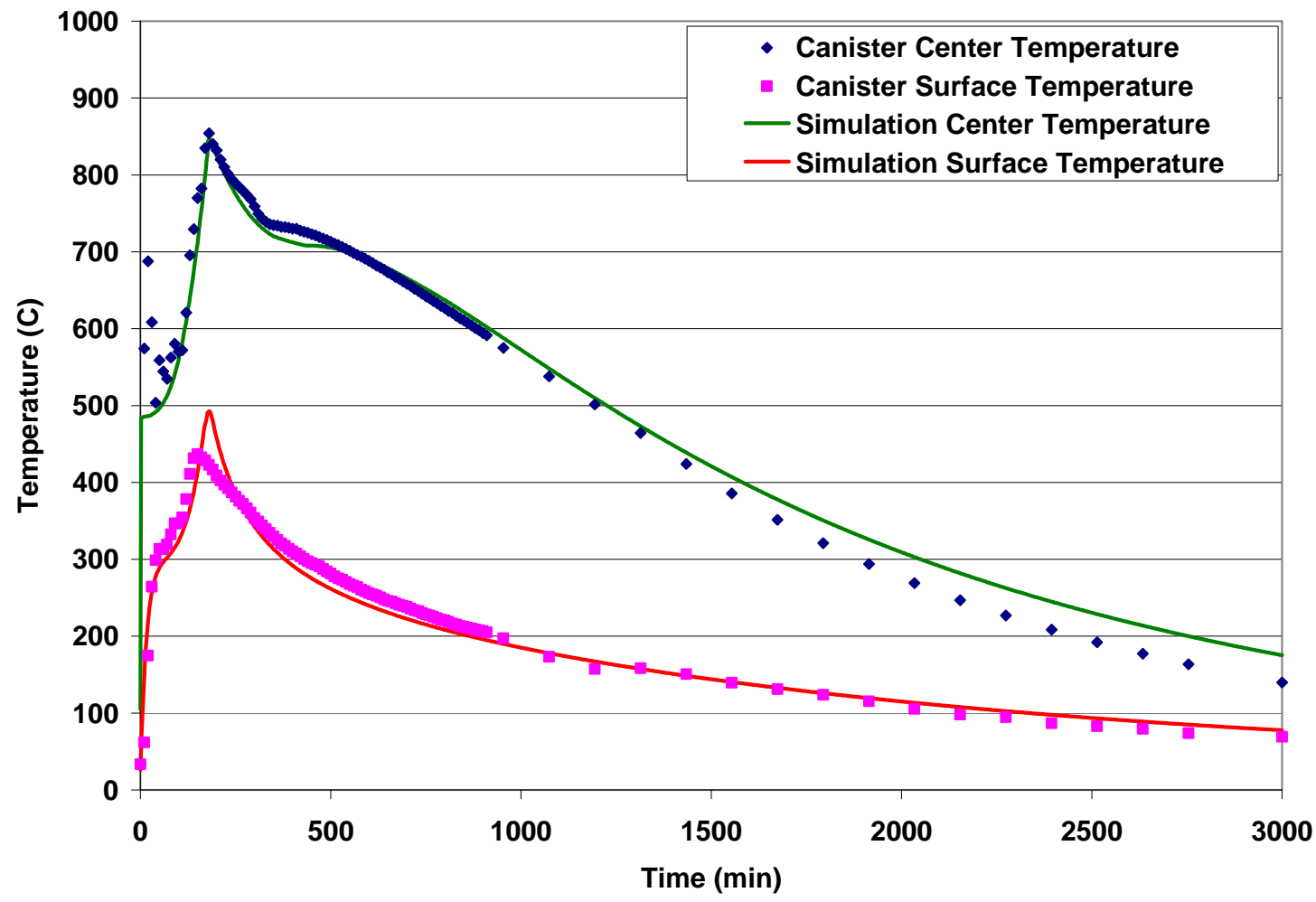

Figure 5 - Temperature curves for thermocouple and simulation data at the 15 inch location of the canister. 


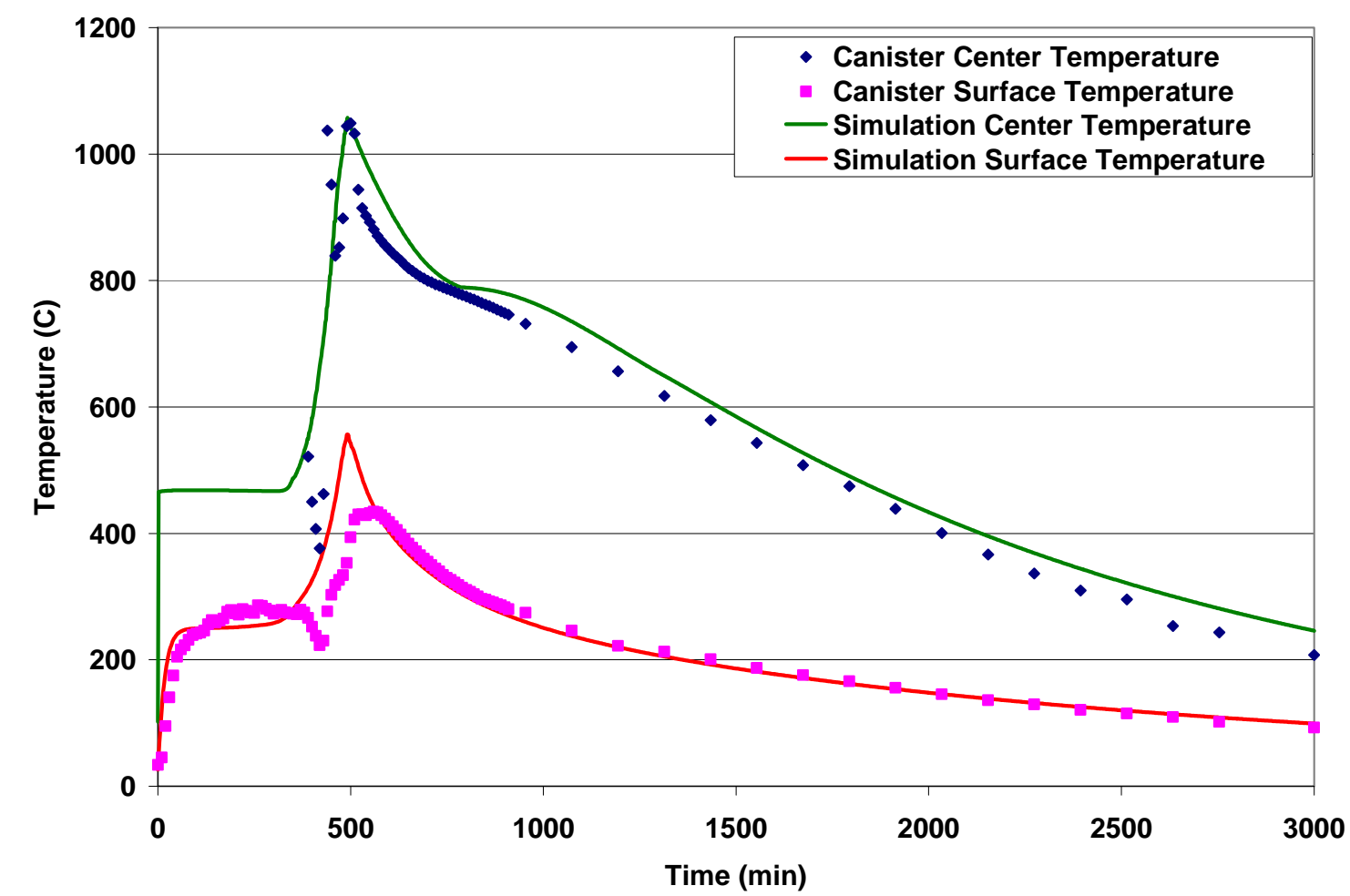

Figure 6 - Temperature curves for thermocouple and simulation data at the 51 inch location of the canister.

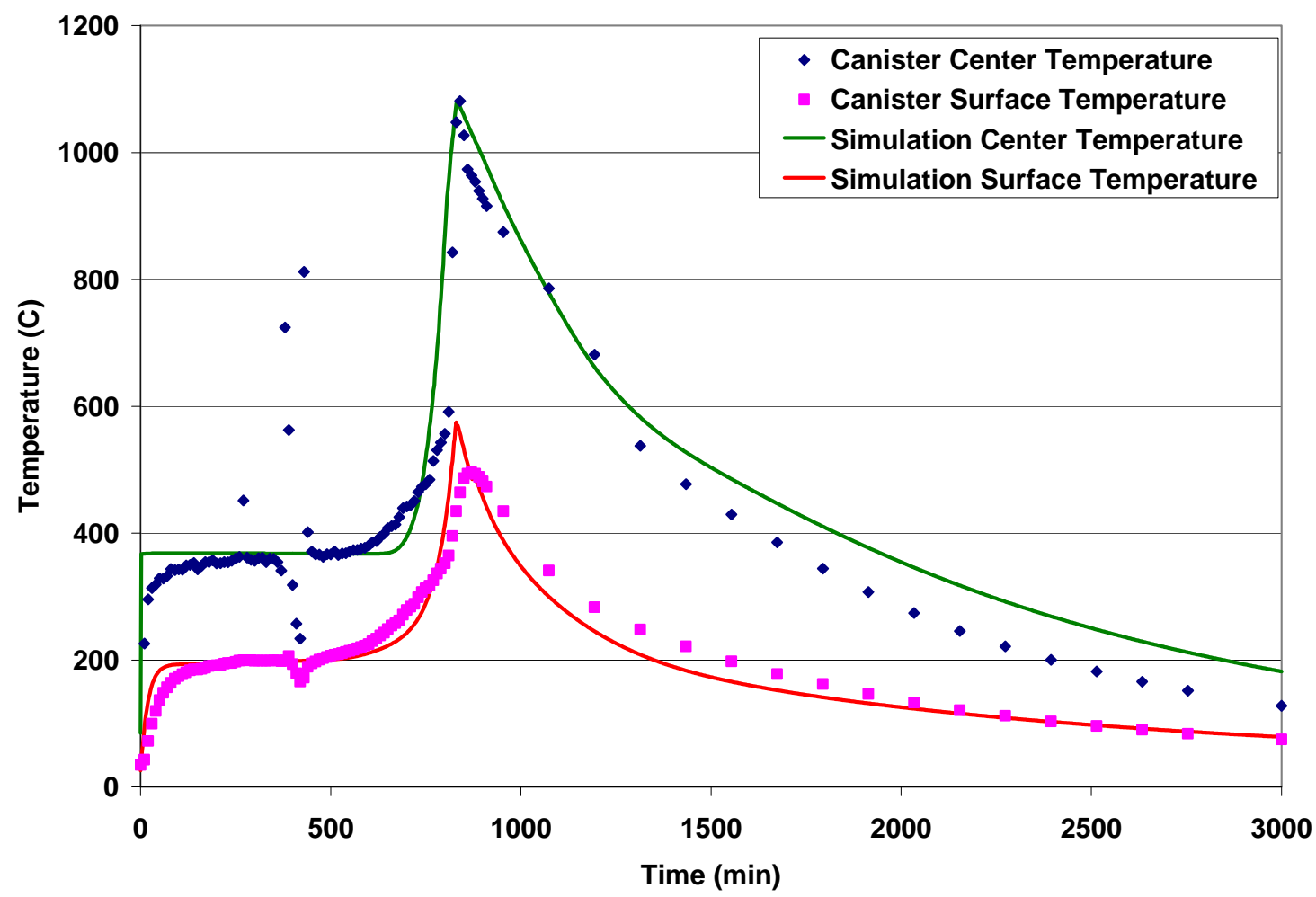

Figure 7 - Temperature curves for thermocouple and simulation data at the 87 inch location of the canister. 


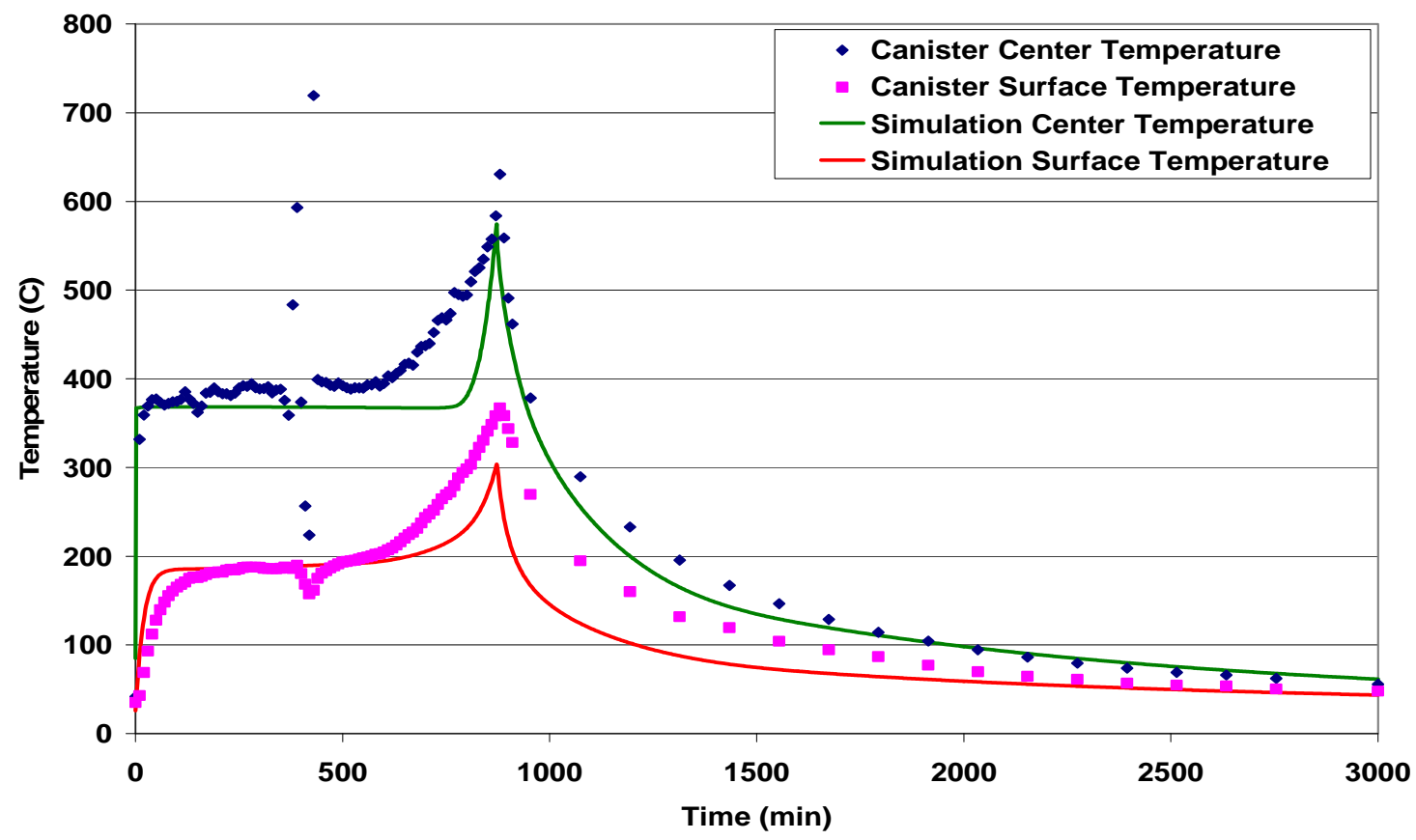

Figure 8 - Temperature curves for thermocouple and simulation data at the 99 inch location of the canister.

\section{Conclusions}

A COMSOL Multiphysics model was developed to predict temperatures of the glass within DWPF canisters during filling and cooldown. The model simulations and experimental data were in good agreement. The largest temperature deviations were $\sim 40^{\circ} \mathrm{C}$ for the 87inch thermocouple location at 3000 minutes and during the initial cooldown at the 51 inch location occurring at approximately 600 minutes. Additionally, the model described in this report predicts the general trends in temperatures during filling and cooling observed experimentally. However, the model was developed using parameters designed to fit a single set of experimental data. Therefore, Q_loss is not currently a function of pour rate and pour temperature. Future work utilizing the existing model should include modifying the Q_loss term to be variable based on flow rate and pour temperature. Further enhancements could include eliminating the Q_loss term for a user defined convection where Navier-Stokes does not need to be solved in order to have convection heat transfer.

\section{References}

1. COMSOL Multiphysics, Version 3.5a, COMSOL Inc, Burlington, MA

2. WSRC-IM-91-116-1, "Chemical Composition Projections for then DWPF Product”, 1995, Revision 1, J.R. Fowler, R.E. Edwards, S.L. Marra, M.J. Plodinec

3. Drawing - W747391, "Savannah River Plant Bldg 221S 200 Area DWPF - CDC Canister Assembly Mechanical”, Revision 0, 1984

4. SRNL-L5200-2009-00013, Interoffice Memorandum, "Predicting Temperatures for AFCI Canistered Waste Glasses”, D.A. Tamburello to J.C. Marra 


\section{Appendix}

\section{Appendix A - Comsol Constants and Expressions}

The following tables are the constants and global expressions directly exported from COMSOL.

COMSOL Constants

\begin{tabular}{|l|l|}
\hline \multicolumn{1}{|c|}{ Constant Name } & \multicolumn{1}{c|}{ Constant Value } \\
\hline Cp_glass & $10[\mathrm{~J} / \mathrm{g} / \mathrm{K}]$ \\
\hline k_glass & $100[\mathrm{~W} / \mathrm{m} / \mathrm{K}]$ \\
\hline gravity & $9.8[\mathrm{~m} / \mathrm{s} \wedge 2]$ \\
\hline R_ig & $.08206[\mathrm{~L} * a t m / \mathrm{mol} / \mathrm{K}]$ \\
\hline MW_air & $28.84[\mathrm{~g} / \mathrm{mol}]$ \\
\hline glass_k_const & $0.6[\mathrm{Btu} / \mathrm{h} / \mathrm{ft} / \mathrm{degF}]$ \\
\hline glass_rho & $167[\mathrm{lb} / \mathrm{ft} \wedge 3]$ \\
\hline glass_cp_const & $0.2[\mathrm{Btu} / \mathrm{lb} / \mathrm{degF}]$ \\
\hline air_k & $0.04[\mathrm{Btu} / \mathrm{h} / \mathrm{ft} / \mathrm{degF}]$ \\
\hline air_cp & $0.26[\mathrm{Btu} / \mathrm{lb} / \mathrm{degF}]$ \\
\hline glass_visc & $10[\mathrm{lb} / \mathrm{h} / \mathrm{ft}]$ \\
\hline air_visc & $0.1[\mathrm{lb} / \mathrm{h} / \mathrm{ft}]$ \\
\hline MW_glass & $2000[\mathrm{~g} / \mathrm{mol}]$ \\
\hline glass_flow_init_const & $228[\mathrm{lb} / \mathrm{h}]$ \\
\hline inlet_area & $0.031416[\mathrm{~m} \wedge 2]$ \\
\hline Lower_area & $0.26512[\mathrm{~m} \wedge 2]$ \\
\hline glass_T_init & $1080[\mathrm{degC}]$ \\
\hline sigma & $5.67 \mathrm{e}-8[\mathrm{~W} / \mathrm{m} \wedge 2 / \mathrm{K} \wedge 4]$ \\
\hline T_atm & $26[\mathrm{degC}]$ \\
\hline Conv_ht & $4[\mathrm{~W} / \mathrm{m} \wedge 2 / \mathrm{K}]$ \\
\hline SS_rho & $494.429[\mathrm{lb} / \mathrm{ft} \wedge 3]$ \\
\hline SS_cp_old & $.135[\mathrm{Btu} / \mathrm{lb} / \mathrm{degF}]$ \\
\hline ContainerHeight & $3[\mathrm{~m}]$ \\
\hline Ta & glass_T_init[1/degC]+32 \\
\hline Tb & 36.98552 \\
\hline Tc & 8.411223 \\
\hline Td & 106.379 \\
\hline Q_loss_base & $320000[\mathrm{~W} / \mathrm{m} / 23]$ \\
\hline dt & $120[\mathrm{~s}]$ \\
\hline GasHeat & $700[\mathrm{~W} / \mathrm{m} / 3]$ \\
\hline FinalPourSwitch & MaxGlass-500[lb] \\
\hline MaxGlass & $3656[\mathrm{lb}]$ \\
\hline MaxHeight & MaxGlass/glass_rho/Lower_area \\
\hline T_Lump_init & $200[\mathrm{degC}]$ \\
\hline int_emmisivity & $0.55[1]$ \\
\hline Max_Gas_temp & $370[\mathrm{degC}]$ \\
\hline Q_Loss_time & $17520[\mathrm{~s}]$ \\
\hline T_amb & $700[\mathrm{degC}]$ \\
\hline T_low & $720[\mathrm{degC}]$ \\
\hline T_high & \\
\hline
\end{tabular}




\section{COMSOL Global Expressions}

\begin{tabular}{|c|c|}
\hline Vaiable Name & Expression \\
\hline Cp_eff & air_cp+glass_cp*(z<Fluid_Height) \\
\hline rho_eff & air_rho+glass_rho_adap*(z<Fluid_Height) \\
\hline T_ramp & $273.15+1[\mathrm{~K} / \mathrm{s}]^{*} \mathrm{t}$ \\
\hline air_rho & 1[atm]/R_ig/T*MW_air \\
\hline Fluid_Height & (Fluid_Height_new $+.01[\mathrm{~m}]) *$ Filling+MaxHeight*(1-Filling) \\
\hline HeatFlow & glass_cp*glass_T_init*glass_flow \\
\hline Fluid_vol & Fluid_Height*Lower_area \\
\hline Q_rad & emmisivity*view_factor*sigma*((T)^4-(T_atm $\left.)^{\wedge} 4\right)+h$ _surface*(T-T_atm) \\
\hline delFluidVol & (Fluid_Height-LastFluidHeight)*Lower_area \\
\hline delHeight & (z<=Fluid_Height)*(z>LastFluidHeight)[1] \\
\hline Q_glass & $(3 *(T$ _glass_top-T)*glass_cp*glass_flow/delFluidVol)*Filling*delHeight*r_factor \\
\hline Q_calc & (Q_glass-Q_loss*(z<Fluid_Height[m]-.01[m])*(z>Fluid_Height[m]-.8)) \\
\hline glass_k & $(0.76449+2.74341 *$ flc2hs(T[1/degC]-950,100))[Btu/h/ft/degF] \\
\hline glass_rho_adap & $\begin{array}{l}(172.31 *(\mathrm{~T}[\mathrm{deg} C]<20)+(\mathrm{T}[\mathrm{degC}]>=20) *(\mathrm{~T}[\mathrm{degC}]<=1200) *(172.2011+.00109 * \mathrm{~T}[1 / \mathrm{degC}]- \\
1.609 \mathrm{e}-5 *(\mathrm{~T}[1 / \mathrm{deg} C])^{\wedge} 2-1.508162 \mathrm{e}-8^{*}(\mathrm{~T}[1 / \mathrm{degC}])^{\wedge} 3+1.3323 \mathrm{e}- \\
\left.\left.11^{*}(\mathrm{~T}[1 / \mathrm{degC}])^{\wedge} 4\right)+151.77 *(\mathrm{~T}[\operatorname{degC}]>1200)\right)[\mathrm{lb} / \mathrm{ft} \wedge 3]\end{array}$ \\
\hline glass_cp & $(0.204+0.16 *$ flc2hs(T[1/degC]-1000,400))[Btu/lb/degF] \\
\hline glass_T & glass_T_init-25[K/ft]*(3[m]-Fluid_Height) \\
\hline Q_rad1 & emmisivity*view_factor*sigma*((T)^4-(T_atm $\left.)^{\wedge} 4\right)$ \\
\hline Conv1 & h_surface*(T-T_atm) \\
\hline SS_k & $\left(-13.589083+3.9578316 * \log \left(\mathrm{T} \_\min [1 / \mathrm{K}]\right)\right)[\mathrm{Btu} / \mathrm{h} / \mathrm{ft} / \mathrm{degF}]$ \\
\hline Conv_inside & h_surface* $(5[\mathrm{~K}])$ \\
\hline Ra_init & gravity*Beta*(T-T_atm)*ContainerHeight^3/(air_visc*air_k/air_rho^2/air_cp) \\
\hline $\mathrm{Ra}$ & Ra_init*(Ra_init>0) \\
\hline Beta & $1 / \mathrm{T}$ \\
\hline $\operatorname{Pr}$ & air_cp*air_visc/air_k \\
\hline h_surface_low & $(\text { air_k/ContainerHeight })^{*}\left(0.68+\left(0.670^{*} \mathrm{Ra}^{\wedge} .25\right) /\left(1.0+(0.492 / \mathrm{Pr})^{\wedge}(9 / 16)\right)^{\wedge}(4 / 9)\right)$ \\
\hline h_surface_high & $\left((\text { air_k/ContainerHeight })^{*}\left(0.825+\left(0.387 * \mathrm{Ra}^{\wedge}(1 / 6)\right) /\left(1.0+(0.492 / \mathrm{Pr})^{\wedge}(9 / 16)\right)^{\wedge}(8 / 27)\right)^{\wedge} 2\right)$ \\
\hline h_surface & (h_surface_low* $(\mathrm{Ra}<=1 \mathrm{e} 9)+\mathrm{h} \_$surface_high*(Ra $\left.\left.>1 \mathrm{e} 9\right)\right)$ \\
\hline T_min & $\mathrm{T}^{*}\left(\mathrm{~T}>\mathrm{T} \_\mathrm{atm}-5\right)+\mathrm{T} \_$atm*(T<=T_atm-5) \\
\hline Internal_rad & $\begin{array}{l}\text { int_emmisivity*view_factor2*sigma*((T_interface })^{\wedge} 4- \\
\left.\left.(\mathrm{T})^{\wedge} 4\right)^{*}(\mathrm{z}>\text { Fluid_Height })^{*} \text { Filling*(T<}<\mathrm{T} \text { _interface }\right)^{*}(0.74-.2 * \mathrm{z}[1 / \mathrm{m}])\end{array}$ \\
\hline T_glass_top & $\left(\mathrm{Ta} /\left((1+\exp (\mathrm{Tb}-\mathrm{Tc} * \text { Fluid_Height[1/ft] }))^{\wedge}(1 / \mathrm{Td})\right)\right)[\mathrm{degC}]^{*}$ Filling \\
\hline TopHeat & (glass_T_init-T_atm)*flc2hs((MaxHeight-(LastFluidHeight+.01[m]))[1/m],.2)+T_atm \\
\hline Filling & $(($ Fluid_Height_new+.01[m])<=MaxHeight)[1] \\
\hline Q_loss & $\begin{array}{l}\left.\text { (Q_loss_base*(-0.0548*(z[1/m] })^{\wedge} 2+0.1045 * z[1 / \mathrm{m}]+0.48\right) *(f l c 2 h s(z[1 / \mathrm{m}]- \\
\left.\text { (Fluid_Height_dummy[1/m]-Q_loss_height[1/m]),(.6568*(z[1/m] })^{\wedge} 2\right)- \\
1.5375 * z[1 / \mathrm{m}]+1.7))) *\left(1 / \exp \left(60 *(r[1 / \mathrm{m}])^{\wedge} 3\right)\right)^{*} \mathrm{Q} \text { _loss_radial }\end{array}$ \\
\hline Q_loss_radial & $\begin{array}{l}(\mathrm{r}[1 / \mathrm{m}]>(0.085 * \mathrm{z}[1 / \mathrm{m}]+0.0685)) *(0.85- \\
.3 * \mathrm{z}[1 / \mathrm{m}]) *(\mathrm{r}<=0.285)+(\mathrm{r}[1 / \mathrm{m}]<=(0.085 * \mathrm{z}[1 / \mathrm{m}]+0.0685))\end{array}$ \\
\hline Fluid_Height_dummy & (Fluid_Height_new+.01[m]) \\
\hline T_interface & (glass_T_init-200[K]) \\
\hline TotalGlassPour & glass_flow_init*t \\
\hline glass_flow & glass_flow_init \\
\hline glass_flux & glass_flow/MW_glass/inlet_area \\
\hline glass_flow_rate & glass_flow/glass_rho/Lower_area \\
\hline view_factor2 & $0.124-0.03 * z[1 / \mathrm{m}]+.15 *(\mathrm{z}[1 / \mathrm{m}]) * \mathrm{flc} 2 \mathrm{hs}((\mathrm{HF} / \mathrm{zF})-.95, .4)$ \\
\hline
\end{tabular}




\begin{tabular}{|c|c|}
\hline Vaiable Name & Expression \\
\hline $\mathrm{HF}$ & Fluid_Height/MaxHeight \\
\hline $\mathrm{zF}$ & z/MaxHeight \\
\hline Q_rad_top & sigma*((glass_T_init)^4-(T)^4)*Filling \\
\hline Gas_Temp & Max_Gas_temp+120*flc2hs(1.5-z[1/m],.5) \\
\hline Q_gas & $\begin{array}{l}(\mathrm{T}<\mathrm{Gas} \text { Temp }) * 2.5 *(\text { Gas_Temp- } \\
\mathrm{T}) * \text { air_cp/(1[s])*air_rho*Filling*(z>(Fluid_Height[m] }))^{*}(\mathrm{r}<0.2[\mathrm{~m}])\end{array}$ \\
\hline Q_loss_height & glass_flow_rate*Q_Loss_time/2.5*z[1/m] \\
\hline view_factor & $1[1]$ \\
\hline emmisivity & $.8[1]$ \\
\hline SS_cp & $(.12+.015 *$ flc2hs(T[1/degF]-452[degF][1/degF],300))[Btu/lb/degF] \\
\hline k_eff & $\begin{array}{l}\text { (30*air_k)*(z>=Fluid_Height)*Filling*(1-flc2hs((HF/zF)-1.0,.1))+glass_k*flc2hs((HF/zF)- } \\
.80,8)^{*} \text { Filling }+ \text { (glass_k*(z<=Fluid_Height)+20*air_k*(z>Fluid_Height))*(1-Filling) }\end{array}$ \\
\hline T_Hold_Center & $\begin{array}{l}((452.2656+240.1336 * z[1 / \mathrm{m}]- \\
\left.\left.40.3449 *(\mathrm{z}[1 / \mathrm{m}])^{\wedge} 2\right)^{*}(\mathrm{z}<=\text { MaxHeight })+289.5 *(\mathrm{z}>\text { MaxHeight })\right)[\operatorname{degC}]\end{array}$ \\
\hline T_Hold_Surface & $\begin{array}{l}\left(\left(148.5466+60.03426 * z[1 / \mathrm{m}]+12.26439 *(z[1 / \mathrm{m}])^{\wedge} 2\right)^{*}(\mathrm{z}<=\text { MaxHeight })+194.8^{*}(\mathrm{z}>\text { MaxHeight })\right)[\mathrm{d} \\
\text { egC }]\end{array}$ \\
\hline T_3inch & $\left(445.1069+187.7072 * z[1 / \mathrm{m}]-13.2499 *(z[1 / \mathrm{m}])^{\wedge 2}\right)[\mathrm{degC}]$ \\
\hline T_6inch & $\left(388.567+125.6953 * z[1 / \mathrm{m}]+20.96399 *(z[1 / \mathrm{m}])^{\wedge} 2\right)[\operatorname{degC}]$ \\
\hline T_9inch & $\left(282.3685+103.7025 * \mathrm{z}[1 / \mathrm{m}]+25.63263 *(\mathrm{z}[1 / \mathrm{m}])^{\wedge} 2\right)[\mathrm{degC}]$ \\
\hline SteadyQ & T_3inch* $(\mathrm{r}<.0764) *(\mathrm{r}>$.076)+T_6inch $*(\mathrm{r}<$.1526)*(r>.1522)+T_9inch* $(\mathrm{r}<.2288) *(\mathrm{r}>$.2284 $)$ \\
\hline glass_flow_init & $\begin{array}{l}(201+120 *(\mathrm{t}>=180 * 60)+40 *(\mathrm{t}>=270 * 60)-170 *(\mathrm{t}>=350 * 60)+70 *(\mathrm{t}>=500 * 60)- \\
20 *(\mathrm{t}>=610 * 60)+20 *(\mathrm{t}>=730+60))[\mathrm{lb} / \mathrm{h}]\end{array}$ \\
\hline glassFlow1 & $201[\mathrm{lb} / \mathrm{h}]^{*} \mathrm{t}(\mathrm{t}<==180[\mathrm{~min}])$ \\
\hline glassFlow2 & $(321.5[\mathrm{lb} / \mathrm{h}] *(\mathrm{t}-180[\mathrm{~min}])+\mathrm{glw} 1) *(\mathrm{t}>180[\mathrm{~min}]) *(\mathrm{t}<=270[\mathrm{~min}])$ \\
\hline glassFlow3 & $(361.7[\mathrm{lb} / \mathrm{h}] *(\mathrm{t}-270[\mathrm{~min}])+\mathrm{glw} 1+\mathrm{glw} 2) *(\mathrm{t}>270[\mathrm{~min}]) *(\mathrm{t}<=350[\mathrm{~min}])$ \\
\hline glassFlow4 & $(192.9[\mathrm{lb} / \mathrm{h}] *(\mathrm{t}-350[\mathrm{~min}])+\mathrm{glw} 1+\mathrm{glw} 2+\mathrm{glw} 3)^{*}(\mathrm{t}>350[\mathrm{~min}]) *(\mathrm{t}<=500[\mathrm{~min}])$ \\
\hline glassFlow5 & $(263.1[\mathrm{lb} / \mathrm{h}] *(\mathrm{t}-500[\mathrm{~min}])+\mathrm{glw} 1+\mathrm{glw} 2+\mathrm{glw} 3+\mathrm{glw} 4) *(\mathrm{t}>500[\mathrm{~min}]) *(\mathrm{t}<=610[\mathrm{~min}])$ \\
\hline glassFlow6 & $\left(241.1[\mathrm{lb} / \mathrm{h}]^{*}(\mathrm{t}-610[\mathrm{~min}])+\mathrm{glw} 1+\mathrm{glw} 2+\mathrm{glw} 3+\mathrm{glw} 4+\mathrm{glw} 5\right) *(\mathrm{t}>610[\mathrm{~min}]) *(\mathrm{t}<=730[\mathrm{~min}])$ \\
\hline glassFlow7 & $\left(263.1[\mathrm{lb} / \mathrm{h}]^{*}(\mathrm{t}-730[\mathrm{~min}])+\mathrm{glw} 1+\mathrm{glw2}+\mathrm{glw3}+\mathrm{glw} 4+\mathrm{glw} 5+\mathrm{glw} 6\right) *(\mathrm{t}>730[\mathrm{~min}])$ \\
\hline glw1 & $201[\mathrm{lb} / \mathrm{h}] * 180[\mathrm{~min}]$ \\
\hline glw2 & $321.5[\mathrm{lb} / \mathrm{h}] * 90[\mathrm{~min}]$ \\
\hline glw3 & $361.7[\mathrm{lb} / \mathrm{h}] * 80[\mathrm{~min}]$ \\
\hline glw4 & $192.9[\mathrm{lb} / \mathrm{h}]^{*} 150[\mathrm{~min}]$ \\
\hline glw5 & $263.1[\mathrm{lb} / \mathrm{h}]^{*} 110[\mathrm{~min}]$ \\
\hline glw6 & $241.1[\mathrm{lb} / \mathrm{h}]^{*} 120[\mathrm{~min}]$ \\
\hline Fluid_Height_new & $\begin{array}{l}\text { (glassFlow1+glassFlow2+glassFlow3+glassFlow4+glassFlow5+glassFlow6+glassFlow7)/glass_r } \\
\text { ho/Lower_area }\end{array}$ \\
\hline glassFlow1_dt & $201[\mathrm{lb} / \mathrm{h}]^{*}(\mathrm{t}-\mathrm{dt}) *(\mathrm{t}<=180[\mathrm{~min}])$ \\
\hline glassFlow2_dt & $\left(321.5[\mathrm{lb} / \mathrm{h}]^{*}(\mathrm{t}-\mathrm{dt}-180[\mathrm{~min}])+\mathrm{glw} 1\right)^{*}(\mathrm{t}>180[\mathrm{~min}]) *(\mathrm{t}<=270[\mathrm{~min}])$ \\
\hline glassFlow3_dt & $(361.7[\mathrm{lb} / \mathrm{h}] *(\mathrm{t}-\mathrm{dt}-270[\mathrm{~min}])+\mathrm{glw} 1+\mathrm{glw} 2) *(\mathrm{t}>270[\mathrm{~min}]) *(\mathrm{t}<=350[\mathrm{~min}])$ \\
\hline glassFlow4_dt & $(192.9[\mathrm{lb} / \mathrm{h}] *(\mathrm{t}-\mathrm{dt}-350[\mathrm{~min}])+\mathrm{glw} 1+\mathrm{glw} 2+\mathrm{glw} 3) *(\mathrm{t}>350[\mathrm{~min}]) *(\mathrm{t}<=500[\mathrm{~min}])$ \\
\hline glassFlow5_dt & $\left(263.1[\mathrm{lb} / \mathrm{h}]^{*}(\mathrm{t}-\mathrm{dt}-500[\mathrm{~min}])+\mathrm{glw} 1+\mathrm{glw} 2+\mathrm{glw} 3+\mathrm{glw} 4\right) *(\mathrm{t}>500[\mathrm{~min}]) *(\mathrm{t}<=610[\mathrm{~min}])$ \\
\hline glassFlow6_dt & $(241.1[\mathrm{lb} / \mathrm{h}] *(\mathrm{t}-\mathrm{dt}-610[\mathrm{~min}])+\mathrm{glw} 1+\mathrm{glw} 2+\mathrm{glw} 3+\mathrm{glw} 4+\mathrm{glw} 5) *(\mathrm{t}>610[\mathrm{~min}]) *(\mathrm{t}<=730[\mathrm{~min}])$ \\
\hline glassFlow7_dt & $\left(263.1[\mathrm{lb} / \mathrm{h}]^{*}(\mathrm{t}-\mathrm{dt}-730[\mathrm{~min}])+\mathrm{glw} 1+\mathrm{glw2}+\mathrm{glw} 3+\mathrm{glw} 4+\mathrm{glw5}+\mathrm{glw6}\right) *(\mathrm{t}>730[\mathrm{~min}])$ \\
\hline LastFluidHeight & $\begin{array}{l}\text { ((glassFlow1_dt+glassFlow2_dt+glassFlow3_dt+glassFlow4_dt+glassFlow5_dt+glassFlow6_dt+ } \\
\text { glassFlow7_dt)/glass_rho/Lower_area) }\end{array}$ \\
\hline r_factor & $1-\mathrm{r}[1 / \mathrm{m}] / 2 * z[1 / \mathrm{m}][1]$ \\
\hline R1_old & flc2hs(T[1/degC]-T_low[1/degC],1)*flc2hs(T_high[1/degC]-T[1/degC],1)*0.001[mol/s/m^3] \\
\hline R1 & $\left(\mathrm{T}>\mathrm{T} \_\right.$low $) *\left(\mathrm{~T}<\mathrm{T} \_\right.$high $) * 0.0001[\mathrm{~mol} / \mathrm{s} / \mathrm{m} \wedge 3]$ \\
\hline
\end{tabular}




\section{Appendix B - Simulation Temperature versus Time Data}

\begin{tabular}{|c|c|c|c|c|c|c|c|c|c|}
\hline $\begin{array}{l}\text { Time } \\
\text { (min) }\end{array}$ & $\begin{array}{l}\text { Bottom } \\
\text { (C) }\end{array}$ & $\begin{array}{c}15 \\
\text { Inch } \\
\text { Center } \\
\text { (C) }\end{array}$ & $\begin{array}{l}15 \text { inch } \\
\text { Surface } \\
\text { (C) }\end{array}$ & $\begin{array}{l}51 \text { inch } \\
\text { Center } \\
\text { (C) }\end{array}$ & $\begin{array}{l}51 \text { inch } \\
\text { Surface } \\
\text { (C) }\end{array}$ & $\begin{array}{l}87 \text { inch } \\
\text { Center } \\
\text { (C) }\end{array}$ & $\begin{array}{l}87 \text { inch } \\
\text { Surface } \\
\text { (C) }\end{array}$ & $\begin{array}{l}99 \text { inch } \\
\text { Center } \\
\text { (C) }\end{array}$ & $\begin{array}{l}99 \text { inch } \\
\text { Surface } \\
\text { (C) }\end{array}$ \\
\hline 0 & 25.9 & 26.0 & 26.0 & 26.0 & 26.0 & 26.0 & 26.0 & 26.0 & 26.0 \\
\hline 10 & 230.1 & 132.5 & 86.9 & 58.3 & 58.5 & 39.9 & 39.7 & 37.4 & 35.3 \\
\hline 20 & 362.9 & 487.8 & 160.3 & 469.4 & 111.7 & 369.2 & 72.4 & 369.2 & 64.4 \\
\hline 30 & 457.5 & 491.5 & 224.8 & 471.5 & 167.6 & 370.0 & 111.6 & 370.0 & 101.6 \\
\hline 40 & 472.8 & 496.8 & 262.3 & 471.5 & 203.1 & 370.0 & 139.5 & 370.0 & 128.4 \\
\hline 50 & 472.7 & 504.2 & 283.3 & 471.5 & 223.9 & 370.0 & 157.9 & 370.0 & 146.6 \\
\hline 60 & 470.3 & 514.0 & 295.9 & 471.5 & 235.6 & 370.0 & 169.7 & 370.0 & 158.6 \\
\hline 70 & 466.9 & 524.5 & 304.9 & 471.5 & 242.1 & 370.0 & 177.0 & 370.0 & 166.4 \\
\hline 80 & 462.9 & 538.4 & 313.0 & 471.5 & 245.8 & 370.0 & 181.6 & 370.0 & 171.4 \\
\hline 90 & 458.5 & 555.2 & 321.8 & 471.5 & 247.8 & 370.0 & 184.5 & 370.0 & 174.5 \\
\hline 100 & 453.8 & 573.9 & 332.2 & 471.5 & 249.0 & 370.0 & 186.2 & 370.0 & 176.5 \\
\hline 110 & 448.9 & 595.2 & 344.6 & 471.5 & 249.7 & 370.0 & 187.3 & 370.0 & 177.7 \\
\hline 120 & 443.9 & 619.2 & 360.0 & 471.5 & 250.2 & 370.0 & 188.0 & 370.0 & 178.4 \\
\hline 130 & 438.7 & 647.8 & 378.0 & 471.5 & 250.5 & 370.0 & 188.4 & 370.0 & 178.9 \\
\hline 140 & 433.4 & 678.9 & 398.6 & 471.5 & 250.8 & 370.0 & 188.7 & 370.0 & 179.2 \\
\hline 150 & 428.1 & 730.3 & 421.7 & 471.5 & 251.1 & 370.0 & 188.9 & 370.0 & 179.5 \\
\hline 160 & 422.8 & 792.7 & 445.1 & 471.5 & 251.4 & 370.0 & 189.1 & 370.0 & 179.6 \\
\hline 170 & 417.5 & 833.5 & 460.1 & 471.5 & 251.7 & 370.0 & 189.2 & 370.0 & 179.7 \\
\hline 180 & 412.2 & 848.2 & 459.9 & 471.5 & 252.1 & 370.0 & 189.3 & 370.0 & 179.8 \\
\hline 190 & 406.9 & 837.3 & 453.8 & 471.4 & 252.5 & 370.0 & 189.5 & 370.0 & 179.9 \\
\hline 200 & 401.8 & 826.0 & 440.0 & 471.4 & 253.0 & 370.0 & 189.6 & 370.0 & 180.0 \\
\hline 210 & 396.8 & 815.3 & 425.8 & 471.4 & 253.6 & 370.0 & 189.8 & 370.0 & 180.2 \\
\hline 220 & 391.9 & 805.2 & 412.6 & 471.4 & 254.2 & 370.0 & 190.0 & 370.0 & 180.3 \\
\hline 230 & 387.1 & 795.7 & 400.5 & 471.4 & 255.0 & 370.0 & 190.2 & 370.0 & 180.5 \\
\hline 240 & 382.5 & 786.7 & 389.7 & 471.4 & 255.7 & 370.0 & 190.4 & 370.0 & 180.7 \\
\hline 250 & 378.0 & 778.3 & 379.8 & 471.4 & 256.6 & 370.0 & 190.7 & 370.0 & 180.9 \\
\hline 260 & 373.7 & 770.6 & 370.9 & 471.3 & 257.4 & 370.0 & 191.0 & 370.0 & 181.1 \\
\hline 270 & 369.5 & 763.4 & 362.7 & 471.3 & 258.5 & 370.0 & 191.3 & 370.0 & 181.3 \\
\hline 280 & 365.5 & 756.5 & 355.2 & 471.3 & 259.7 & 370.0 & 191.6 & 370.0 & 181.6 \\
\hline 290 & 361.6 & 750.3 & 348.2 & 471.3 & 261.4 & 370.0 & 192.0 & 370.0 & 181.9 \\
\hline 300 & 358.0 & 744.7 & 341.8 & 471.3 & 263.6 & 369.9 & 192.4 & 370.0 & 182.2 \\
\hline 310 & 355.4 & 739.6 & 335.7 & 471.4 & 266.6 & 369.9 & 192.8 & 369.9 & 182.6 \\
\hline 320 & 353.0 & 735.1 & 330.1 & 471.9 & 270.4 & 369.9 & 193.3 & 369.9 & 183.0 \\
\hline 330 & 350.5 & 731.0 & 324.8 & 474.7 & 275.1 & 369.9 & 193.8 & 369.9 & 183.4 \\
\hline 340 & 348.1 & 727.5 & 319.8 & 481.6 & 281.1 & 369.9 & 194.3 & 369.9 & 183.8 \\
\hline 350 & 345.6 & 724.4 & 315.2 & 494.5 & 288.7 & 369.9 & 194.8 & 369.9 & 184.2 \\
\hline 360 & 343.1 & 722.4 & 310.8 & 504.8 & 297.2 & 369.9 & 195.3 & 369.9 & 184.6 \\
\hline 370 & 340.6 & 720.6 & 306.7 & 519.2 & 305.3 & 369.9 & 195.8 & 369.9 & 185.0 \\
\hline 380 & 338.2 & 718.9 & 302.8 & 536.9 & 313.5 & 369.9 & 196.1 & 369.9 & 185.3 \\
\hline 390 & 335.7 & 717.3 & 299.1 & 557.7 & 322.7 & 369.9 & 196.5 & 369.9 & 185.6 \\
\hline 400 & 333.2 & 715.9 & 295.6 & 583.0 & 333.5 & 369.9 & 196.9 & 369.9 & 185.9 \\
\hline
\end{tabular}




\begin{tabular}{|c|c|c|c|c|c|c|c|c|c|}
\hline $\begin{array}{l}\text { Time } \\
\text { (min) }\end{array}$ & $\begin{array}{l}\text { Bottom } \\
\text { (C) }\end{array}$ & $\begin{array}{c}15 \\
\text { Inch } \\
\text { Center } \\
\text { (C) }\end{array}$ & $\begin{array}{l}15 \text { inch } \\
\text { Surface } \\
\text { (C) }\end{array}$ & $\begin{array}{l}51 \text { inch } \\
\text { Center } \\
\text { (C) }\end{array}$ & $\begin{array}{c}51 \text { inch } \\
\text { Surface } \\
\text { (C) }\end{array}$ & $\begin{array}{l}87 \text { inch } \\
\text { Center } \\
\text { (C) }\end{array}$ & $\begin{array}{c}87 \text { inch } \\
\text { Surface } \\
\text { (C) }\end{array}$ & $\begin{array}{l}99 \text { inch } \\
\text { Center } \\
\text { (C) }\end{array}$ & $\begin{array}{l}99 \text { inch } \\
\text { Surface } \\
\text { (C) }\end{array}$ \\
\hline 410 & 330.7 & 715.6 & 292.2 & 616.7 & 346.0 & 369.9 & 197.2 & 369.9 & 186.2 \\
\hline 420 & 328.3 & 715.7 & 289.1 & 654.8 & 360.8 & 369.9 & 197.5 & 369.9 & 186.5 \\
\hline 430 & 325.8 & 715.7 & 286.1 & 699.2 & 378.5 & 369.9 & 197.9 & 369.9 & 186.8 \\
\hline 440 & 323.4 & 715.5 & 283.2 & 765.4 & 399.3 & 369.9 & 198.3 & 369.9 & 187.1 \\
\hline 450 & 320.9 & 715.2 & 280.4 & 857.4 & 424.5 & 369.9 & 198.6 & 369.9 & 187.4 \\
\hline 460 & 318.5 & 714.8 & 277.7 & 953.9 & 452.7 & 369.9 & 199.1 & 369.9 & 187.7 \\
\hline 470 & 316.1 & 714.2 & 275.1 & 1017.0 & 481.2 & 369.9 & 199.5 & 369.9 & 188.0 \\
\hline 480 & 313.7 & 713.4 & 272.5 & 1050.6 & 501.6 & 369.9 & 200.0 & 369.9 & 188.3 \\
\hline 490 & 311.3 & 712.5 & 270.1 & 1056.6 & 506.0 & 369.9 & 200.5 & 369.9 & 188.6 \\
\hline 500 & 308.9 & 711.5 & 267.7 & 1040.5 & 496.3 & 369.9 & 201.1 & 369.9 & 188.9 \\
\hline 510 & 306.6 & 710.3 & 265.4 & 1024.7 & 486.0 & 369.9 & 201.9 & 369.9 & 189.3 \\
\hline 520 & 304.2 & 709.0 & 263.2 & 1009.4 & 473.3 & 369.9 & 202.8 & 369.9 & 189.7 \\
\hline 530 & 301.9 & 707.5 & 261.0 & 994.8 & 460.7 & 369.9 & 203.9 & 369.9 & 190.1 \\
\hline 540 & 299.6 & 705.9 & 258.9 & 980.7 & 449.0 & 369.9 & 205.1 & 369.9 & 190.6 \\
\hline 550 & 297.3 & 704.2 & 256.8 & 967.1 & 438.2 & 369.9 & 206.6 & 369.9 & 191.2 \\
\hline 560 & 295.1 & 702.4 & 254.8 & 954.0 & 428.3 & 369.9 & 208.2 & 369.9 & 191.8 \\
\hline 570 & 292.8 & 700.5 & 252.8 & 941.3 & 419.2 & 369.9 & 210.0 & 369.9 & 192.6 \\
\hline 580 & 290.6 & 698.5 & 250.9 & 928.8 & 410.8 & 369.9 & 212.0 & 369.9 & 193.4 \\
\hline 590 & 288.4 & 696.4 & 249.0 & 916.5 & 403.0 & 369.9 & 214.1 & 369.9 & 194.3 \\
\hline 600 & 286.2 & 694.1 & 247.2 & 904.5 & 395.7 & 370.0 & 216.5 & 369.9 & 195.3 \\
\hline 610 & 284.0 & 691.8 & 245.3 & 892.6 & 388.8 & 370.2 & 219.0 & 369.9 & 196.4 \\
\hline 620 & 281.8 & 689.5 & 243.6 & 881.6 & 382.3 & 370.8 & 221.7 & 369.9 & 197.6 \\
\hline 630 & 279.7 & 687.0 & 241.9 & 870.9 & 376.2 & 371.8 & 224.4 & 369.9 & 198.9 \\
\hline 640 & 277.6 & 684.5 & 240.2 & 860.5 & 370.3 & 373.5 & 227.4 & 369.9 & 200.2 \\
\hline 650 & 275.5 & 681.9 & 238.5 & 850.6 & 364.7 & 376.2 & 230.6 & 369.9 & 201.7 \\
\hline 660 & 273.4 & 679.2 & 236.9 & 841.4 & 359.4 & 380.2 & 234.1 & 369.9 & 203.2 \\
\hline 670 & 271.3 & 676.5 & 235.3 & 832.8 & 354.2 & 386.1 & 237.9 & 369.9 & 204.9 \\
\hline 680 & 269.3 & 673.7 & 233.7 & 824.8 & 349.3 & 394.4 & 242.3 & 369.9 & 206.7 \\
\hline 690 & 267.3 & 670.9 & 232.2 & 817.3 & 344.5 & 405.3 & 247.2 & 369.9 & 208.5 \\
\hline 700 & 265.3 & 668.0 & 230.6 & 810.5 & 340.0 & 419.9 & 252.8 & 369.9 & 210.5 \\
\hline 710 & 263.3 & 665.2 & 229.2 & 804.3 & 335.6 & 439.1 & 259.4 & 369.9 & 212.5 \\
\hline 720 & 261.3 & 662.3 & 227.7 & 798.6 & 331.3 & 462.7 & 267.1 & 370.0 & 214.7 \\
\hline 730 & 259.4 & 659.3 & 226.2 & 793.4 & 327.2 & 491.9 & 276.1 & 370.2 & 217.0 \\
\hline 740 & 257.5 & 656.4 & 224.8 & 788.7 & 323.3 & 525.4 & 286.4 & 370.6 & 219.4 \\
\hline 750 & 255.6 & 653.5 & 223.4 & 784.5 & 319.5 & 567.8 & 298.3 & 371.5 & 222.1 \\
\hline 760 & 253.7 & 650.6 & 222.1 & 780.9 & 315.9 & 621.8 & 312.1 & 373.2 & 224.9 \\
\hline 770 & 251.8 & 647.6 & 220.7 & 779.7 & 312.4 & 687.0 & 328.7 & 375.9 & 228.0 \\
\hline 780 & 249.9 & 644.6 & 219.4 & 779.6 & 309.1 & 766.3 & 348.6 & 380.0 & 231.4 \\
\hline 790 & 248.1 & 641.5 & 218.1 & 779.4 & 305.9 & 861.5 & 373.1 & 386.0 & 235.2 \\
\hline 800 & 246.3 & 638.4 & 216.8 & 779.1 & 302.9 & 955.1 & 404.4 & 394.7 & 239.4 \\
\hline 810 & 244.5 & 635.2 & 215.5 & 778.7 & 299.9 & 1024.7 & 443.2 & 407.4 & 244.3 \\
\hline 820 & 242.7 & 632.1 & 214.2 & 778.2 & 297.0 & 1074.7 & 481.2 & 424.2 & 250.1 \\
\hline 830 & 240.9 & 628.9 & 213.0 & 777.6 & 294.2 & 1093.7 & 498.3 & 446.0 & 257.0 \\
\hline 840 & 239.2 & 625.7 & 211.8 & 776.9 & 291.5 & 1076.2 & 489.4 & 473.2 & 265.1 \\
\hline 850 & 237.5 & 622.4 & 210.6 & 776.1 & 288.9 & 1058.4 & 475.1 & 508.0 & 274.8 \\
\hline 860 & 235.8 & 619.1 & 209.4 & 775.1 & 286.4 & 1040.8 & 461.0 & 553.3 & 286.4 \\
\hline 870 & 234.1 & 615.9 & 208.2 & 774.0 & 283.9 & 1023.6 & 448.1 & 606.5 & 300.3 \\
\hline
\end{tabular}




\begin{tabular}{|c|c|c|c|c|c|c|c|c|c|}
\hline $\begin{array}{l}\text { Time } \\
\text { (min) }\end{array}$ & $\begin{array}{l}\text { Bottom } \\
\text { (C) }\end{array}$ & $\begin{array}{c}15 \\
\text { Inch } \\
\text { Center } \\
\text { (C) }\end{array}$ & $\begin{array}{l}15 \text { inch } \\
\text { Surface } \\
\text { (C) }\end{array}$ & $\begin{array}{l}51 \text { inch } \\
\text { Center } \\
\text { (C) }\end{array}$ & $\begin{array}{l}51 \text { inch } \\
\text { Surface } \\
\text { (C) }\end{array}$ & $\begin{array}{l}87 \text { inch } \\
\text { Center } \\
\text { (C) }\end{array}$ & $\begin{array}{l}87 \text { inch } \\
\text { Surface } \\
\text { (C) }\end{array}$ & $\begin{array}{l}99 \text { inch } \\
\text { Center } \\
\text { (C) }\end{array}$ & $\begin{array}{c}99 \text { inch } \\
\text { Surface } \\
\text { (C) }\end{array}$ \\
\hline 880 & 232.4 & 612.6 & 207.0 & 772.8 & 281.5 & 1006.7 & 447.3 & 593.7 & 329.4 \\
\hline 890 & 230.7 & 609.3 & 205.9 & 771.5 & 279.1 & 990.4 & 439.1 & 580.5 & 340.0 \\
\hline 900 & 229.1 & 605.9 & 204.8 & 770.0 & 276.8 & 974.2 & 428.7 & 564.9 & 338.6 \\
\hline 910 & 227.4 & 602.6 & 203.6 & 768.4 & 274.6 & 957.9 & 418.2 & 548.4 & 333.0 \\
\hline 920 & 225.8 & 599.2 & 202.5 & 766.7 & 272.5 & 941.7 & 408.1 & 531.7 & 325.9 \\
\hline 930 & 224.2 & 595.9 & 201.4 & 764.9 & 270.3 & 925.8 & 398.6 & 515.2 & 318.3 \\
\hline 940 & 222.7 & 592.5 & 200.4 & 763.0 & 268.3 & 910.3 & 389.5 & 499.7 & 310.5 \\
\hline 950 & 221.1 & 589.2 & 199.3 & 760.9 & 266.3 & 895.1 & 381.0 & 485.3 & 302.8 \\
\hline 960 & 219.5 & 585.8 & 198.2 & 758.8 & 264.3 & 880.2 & 372.8 & 471.8 & 295.1 \\
\hline 970 & 218.0 & 582.4 & 197.2 & 756.5 & 262.4 & 865.5 & 365.1 & 459.0 & 287.6 \\
\hline 980 & 216.5 & 579.1 & 196.1 & 754.1 & 260.5 & 851.1 & 357.7 & 446.7 & 280.2 \\
\hline 990 & 215.0 & 575.7 & 195.1 & 751.7 & 258.7 & 836.8 & 350.6 & 434.8 & 272.9 \\
\hline 1000 & 213.5 & 572.3 & 194.1 & 749.1 & 256.9 & 822.8 & 343.8 & 423.3 & 265.8 \\
\hline 1010 & 212.0 & 569.0 & 193.1 & 746.5 & 255.1 & 808.9 & 337.3 & 412.2 & 258.7 \\
\hline 1020 & 210.5 & 565.6 & 192.1 & 743.8 & 253.4 & 795.3 & 331.0 & 401.4 & 251.8 \\
\hline 1030 & 209.1 & 562.3 & 191.1 & 741.0 & 251.7 & 781.9 & 325.0 & 390.9 & 245.0 \\
\hline 1040 & 207.7 & 558.9 & 190.1 & 738.1 & 250.1 & 768.7 & 319.1 & 380.8 & 238.3 \\
\hline 1050 & 206.2 & 555.6 & 189.2 & 735.1 & 248.5 & 755.8 & 313.5 & 370.9 & 231.8 \\
\hline 1060 & 204.8 & 552.2 & 188.2 & 732.1 & 246.9 & 743.1 & 308.0 & 361.5 & 225.3 \\
\hline 1070 & 203.4 & 548.9 & 187.3 & 729.1 & 245.3 & 730.6 & 302.7 & 352.3 & 219.0 \\
\hline 1080 & 202.1 & 545.6 & 186.3 & 725.9 & 243.8 & 718.3 & 297.5 & 343.4 & 212.8 \\
\hline 1090 & 200.7 & 542.3 & 185.4 & 722.8 & 242.3 & 706.3 & 292.5 & 334.7 & 206.7 \\
\hline 1100 & 199.4 & 539.0 & 184.5 & 719.5 & 240.8 & 694.6 & 287.7 & 326.4 & 200.8 \\
\hline 1110 & 198.0 & 535.7 & 183.6 & 716.2 & 239.4 & 683.2 & 283.0 & 318.2 & 195.0 \\
\hline 1120 & 196.7 & 532.4 & 182.7 & 712.9 & 237.9 & 672.0 & 278.4 & 310.3 & 189.3 \\
\hline 1130 & 195.4 & 529.1 & 181.8 & 709.6 & 236.5 & 661.1 & 274.0 & 302.6 & 183.7 \\
\hline 1140 & 194.1 & 525.8 & 180.9 & 706.2 & 235.2 & 651.0 & 269.6 & 295.2 & 178.3 \\
\hline 1150 & 192.8 & 522.6 & 180.0 & 702.7 & 233.8 & 641.6 & 265.4 & 288.0 & 173.1 \\
\hline 1160 & 191.5 & 519.4 & 179.1 & 699.3 & 232.5 & 632.5 & 261.4 & 280.9 & 167.9 \\
\hline 1170 & 190.3 & 516.1 & 178.3 & 695.8 & 231.1 & 623.8 & 257.4 & 274.2 & 162.9 \\
\hline 1180 & 189.0 & 512.9 & 177.4 & 692.3 & 229.9 & 615.3 & 253.5 & 267.6 & 158.0 \\
\hline 1190 & 187.8 & 509.7 & 176.5 & 688.8 & 228.6 & 607.1 & 249.8 & 261.2 & 153.3 \\
\hline 1200 & 186.5 & 506.6 & 175.7 & 685.3 & 227.3 & 599.2 & 246.1 & 255.1 & 148.8 \\
\hline 1210 & 185.3 & 503.4 & 174.9 & 681.8 & 226.1 & 591.6 & 242.6 & 249.2 & 144.3 \\
\hline 1220 & 184.1 & 500.3 & 174.0 & 678.2 & 224.9 & 584.3 & 239.2 & 243.4 & 140.0 \\
\hline 1230 & 182.9 & 497.1 & 173.2 & 674.7 & 223.6 & 577.2 & 235.8 & 237.9 & 135.9 \\
\hline 1240 & 181.7 & 494.0 & 172.4 & 671.2 & 222.5 & 570.4 & 232.6 & 232.6 & 131.9 \\
\hline 1250 & 180.6 & 490.9 & 171.6 & 667.7 & 221.3 & 563.8 & 229.4 & 227.5 & 128.1 \\
\hline 1260 & 179.4 & 487.8 & 170.8 & 664.2 & 220.1 & 557.5 & 226.4 & 222.6 & 124.4 \\
\hline 1270 & 178.3 & 484.7 & 169.9 & 660.7 & 219.0 & 551.5 & 223.4 & 217.8 & 120.8 \\
\hline 1280 & 177.1 & 481.7 & 169.2 & 657.3 & 217.8 & 545.6 & 220.6 & 213.3 & 117.4 \\
\hline 1290 & 176.0 & 478.6 & 168.4 & 654.0 & 216.7 & 540.1 & 217.8 & 208.9 & 114.1 \\
\hline 1300 & 174.9 & 475.6 & 167.6 & 650.8 & 215.6 & 534.7 & 215.1 & 204.7 & 111.0 \\
\hline 1310 & 173.8 & 472.6 & 166.8 & 647.6 & 214.5 & 529.6 & 212.5 & 200.7 & 108.0 \\
\hline 1320 & 172.7 & 469.6 & 166.0 & 644.2 & 213.5 & 524.6 & 209.9 & 196.9 & 105.2 \\
\hline 1330 & 171.6 & 466.7 & 165.3 & 640.9 & 212.4 & 519.9 & 207.5 & 193.2 & 102.5 \\
\hline 1340 & 170.5 & 463.7 & 164.5 & 637.5 & 211.3 & 515.4 & 205.1 & 189.7 & 99.9 \\
\hline
\end{tabular}




\begin{tabular}{|c|c|c|c|c|c|c|c|c|c|}
\hline $\begin{array}{l}\text { Time } \\
(\min )\end{array}$ & $\begin{array}{l}\text { Bottom } \\
\text { (C) }\end{array}$ & $\begin{array}{c}15 \\
\text { Inch } \\
\text { Center } \\
\text { (C) }\end{array}$ & $\begin{array}{l}15 \text { inch } \\
\text { Surface } \\
\text { (C) }\end{array}$ & $\begin{array}{l}51 \text { inch } \\
\text { Center } \\
\text { (C) }\end{array}$ & $\begin{array}{l}51 \text { inch } \\
\text { Surface } \\
\text { (C) }\end{array}$ & $\begin{array}{l}87 \text { inch } \\
\text { Center } \\
\text { (C) }\end{array}$ & $\begin{array}{l}87 \text { inch } \\
\text { Surface } \\
\text { (C) }\end{array}$ & $\begin{array}{l}99 \text { inch } \\
\text { Center } \\
\text { (C) }\end{array}$ & $\begin{array}{c}99 \text { inch } \\
\text { Surface } \\
\text { (C) }\end{array}$ \\
\hline 1350 & 169.4 & 460.8 & 163.7 & 634.2 & 210.3 & 511.0 & 202.8 & 186.4 & 97.4 \\
\hline 1360 & 168.4 & 457.9 & 163.0 & 630.8 & 209.3 & 506.9 & 200.6 & 183.2 & 95.1 \\
\hline 1370 & 167.3 & 455.0 & 162.2 & 627.4 & 208.2 & 502.8 & 198.4 & 180.2 & 92.9 \\
\hline 1380 & 166.3 & 452.1 & 161.5 & 624.0 & 207.2 & 499.0 & 196.3 & 177.3 & 90.8 \\
\hline 1390 & 165.3 & 449.2 & 160.8 & 620.6 & 206.2 & 495.3 & 194.3 & 174.6 & 88.9 \\
\hline 1400 & 164.3 & 446.4 & 160.0 & 617.2 & 205.3 & 491.8 & 192.4 & 172.0 & 87.0 \\
\hline 1410 & 163.3 & 443.5 & 159.3 & 613.8 & 204.3 & 488.3 & 190.5 & 169.5 & 85.3 \\
\hline 1420 & 162.3 & 440.7 & 158.6 & 610.4 & 203.3 & 485.0 & 188.7 & 167.2 & 83.6 \\
\hline 1430 & 161.3 & 437.9 & 157.9 & 607.0 & 202.3 & 481.8 & 186.9 & 165.0 & 82.1 \\
\hline 1440 & 160.3 & 435.1 & 157.1 & 603.6 & 201.4 & 478.8 & 185.2 & 162.9 & 80.7 \\
\hline 1450 & 159.3 & 432.4 & 156.4 & 600.2 & 200.5 & 475.8 & 183.5 & 160.9 & 79.3 \\
\hline 1460 & 158.3 & 429.6 & 155.7 & 596.8 & 199.5 & 472.9 & 181.9 & 159.0 & 78.1 \\
\hline 1470 & 157.4 & 426.9 & 155.0 & 593.4 & 198.6 & 470.0 & 180.4 & 157.2 & 76.9 \\
\hline 1480 & 156.4 & 424.2 & 154.3 & 590.1 & 197.7 & 467.3 & 178.9 & 155.5 & 75.8 \\
\hline 1490 & 155.5 & 421.5 & 153.7 & 586.7 & 196.8 & 464.6 & 177.4 & 153.9 & 74.8 \\
\hline 1500 & 154.6 & 418.9 & 153.0 & 583.3 & 195.9 & 461.9 & 176.0 & 152.4 & 73.9 \\
\hline 1510 & 153.6 & 416.2 & 152.3 & 580.0 & 195.0 & 459.3 & 174.6 & 151.0 & 73.0 \\
\hline 1520 & 152.7 & 413.6 & 151.6 & 576.7 & 194.1 & 456.7 & 173.3 & 149.6 & 72.2 \\
\hline 1530 & 151.8 & 411.0 & 150.9 & 573.3 & 193.2 & 454.2 & 172.0 & 148.4 & 71.5 \\
\hline 1540 & 150.9 & 408.4 & 150.3 & 570.0 & 192.4 & 451.7 & 170.7 & 147.2 & 70.9 \\
\hline 1550 & 150.0 & 405.8 & 149.6 & 566.7 & 191.5 & 449.2 & 169.5 & 146.1 & 70.3 \\
\hline 1560 & 149.2 & 403.2 & 148.9 & 563.4 & 190.7 & 446.7 & 168.3 & 145.0 & 69.8 \\
\hline 1570 & 148.3 & 400.7 & 148.3 & 560.2 & 189.8 & 444.2 & 167.1 & 144.0 & 69.3 \\
\hline 1580 & 147.4 & 398.1 & 147.6 & 556.9 & 189.0 & 441.8 & 166.0 & 143.0 & 68.8 \\
\hline 1590 & 146.6 & 395.6 & 147.0 & 553.7 & 188.1 & 439.3 & 164.9 & 142.1 & 68.4 \\
\hline 1600 & 145.7 & 393.1 & 146.3 & 550.4 & 187.3 & 436.9 & 163.8 & 141.1 & 68.0 \\
\hline 1610 & 144.9 & 390.7 & 145.7 & 547.2 & 186.5 & 434.4 & 162.7 & 140.3 & 67.6 \\
\hline 1620 & 144.0 & 388.2 & 145.1 & 544.0 & 185.7 & 431.9 & 161.7 & 139.4 & 67.3 \\
\hline 1630 & 143.2 & 385.7 & 144.4 & 540.8 & 184.9 & 429.5 & 160.7 & 138.6 & 66.9 \\
\hline 1640 & 142.4 & 383.3 & 143.8 & 537.6 & 184.1 & 427.0 & 159.7 & 137.8 & 66.6 \\
\hline 1650 & 141.6 & 380.9 & 143.2 & 534.5 & 183.3 & 424.6 & 158.7 & 136.9 & 66.2 \\
\hline 1660 & 140.7 & 378.5 & 142.6 & 531.3 & 182.5 & 422.1 & 157.7 & 136.2 & 65.9 \\
\hline 1670 & 139.9 & 376.1 & 141.9 & 528.2 & 181.7 & 419.7 & 156.8 & 135.4 & 65.6 \\
\hline 1680 & 139.1 & 373.8 & 141.3 & 525.1 & 180.9 & 417.3 & 155.9 & 134.6 & 65.3 \\
\hline 1690 & 138.4 & 371.5 & 140.7 & 522.0 & 180.2 & 414.8 & 155.0 & 133.9 & 65.0 \\
\hline 1700 & 137.6 & 369.1 & 140.1 & 518.9 & 179.4 & 412.4 & 154.1 & 133.1 & 64.7 \\
\hline 1710 & 136.8 & 366.8 & 139.5 & 515.9 & 178.6 & 410.0 & 153.2 & 132.4 & 64.5 \\
\hline 1720 & 136.0 & 364.5 & 138.9 & 512.8 & 177.9 & 407.6 & 152.3 & 131.6 & 64.2 \\
\hline 1730 & 135.3 & 362.3 & 138.3 & 509.8 & 177.1 & 405.2 & 151.4 & 130.9 & 63.9 \\
\hline 1740 & 134.5 & 360.0 & 137.7 & 506.8 & 176.4 & 402.8 & 150.6 & 130.2 & 63.7 \\
\hline 1750 & 133.8 & 357.8 & 137.1 & 503.8 & 175.6 & 400.4 & 149.8 & 129.4 & 63.4 \\
\hline 1760 & 133.0 & 355.5 & 136.5 & 500.8 & 174.9 & 398.0 & 148.9 & 128.7 & 63.2 \\
\hline 1770 & 132.3 & 353.3 & 136.0 & 497.8 & 174.2 & 395.6 & 148.1 & 128.0 & 62.9 \\
\hline 1780 & 131.6 & 351.1 & 135.4 & 494.9 & 173.4 & 393.2 & 147.3 & 127.3 & 62.7 \\
\hline 1790 & 130.8 & 348.9 & 134.8 & 492.0 & 172.7 & 390.8 & 146.5 & 126.6 & 62.4 \\
\hline 1800 & 130.1 & 346.8 & 134.2 & 489.1 & 172.0 & 388.5 & 145.8 & 125.9 & 62.2 \\
\hline 1810 & 129.4 & 344.6 & 133.7 & 486.2 & 171.3 & 386.1 & 145.0 & 125.2 & 61.9 \\
\hline
\end{tabular}




\begin{tabular}{|c|c|c|c|c|c|c|c|c|c|}
\hline $\begin{array}{l}\text { Time } \\
(\min )\end{array}$ & $\begin{array}{l}\text { Bottom } \\
\text { (C) }\end{array}$ & $\begin{array}{c}15 \\
\text { Inch } \\
\text { Center } \\
\text { (C) }\end{array}$ & $\begin{array}{l}15 \text { inch } \\
\text { Surface } \\
\text { (C) }\end{array}$ & $\begin{array}{l}51 \text { inch } \\
\text { Center } \\
\text { (C) }\end{array}$ & $\begin{array}{l}51 \text { inch } \\
\text { Surface } \\
\text { (C) }\end{array}$ & $\begin{array}{l}87 \text { inch } \\
\text { Center } \\
\text { (C) }\end{array}$ & $\begin{array}{l}87 \text { inch } \\
\text { Surface } \\
\text { (C) }\end{array}$ & $\begin{array}{l}99 \text { inch } \\
\text { Center } \\
\text { (C) }\end{array}$ & $\begin{array}{c}99 \text { inch } \\
\text { Surface } \\
\text { (C) }\end{array}$ \\
\hline 1820 & 128.7 & 342.5 & 133.1 & 483.3 & 170.6 & 383.8 & 144.2 & 124.5 & 61.7 \\
\hline 1830 & 128.0 & 340.4 & 132.5 & 480.5 & 169.9 & 381.4 & 143.5 & 123.8 & 61.5 \\
\hline 1840 & 127.3 & 338.3 & 132.0 & 477.6 & 169.2 & 379.1 & 142.7 & 123.1 & 61.2 \\
\hline 1850 & 126.6 & 336.2 & 131.4 & 474.8 & 168.5 & 376.8 & 142.0 & 122.5 & 61.0 \\
\hline 1860 & 125.9 & 334.1 & 130.9 & 472.0 & 167.8 & 374.5 & 141.3 & 121.8 & 60.8 \\
\hline 1870 & 125.3 & 332.1 & 130.3 & 469.2 & 167.1 & 372.2 & 140.5 & 121.1 & 60.6 \\
\hline 1880 & 124.6 & 330.0 & 129.8 & 466.4 & 166.4 & 369.9 & 139.8 & 120.5 & 60.3 \\
\hline 1890 & 123.9 & 328.0 & 129.2 & 463.7 & 165.7 & 367.6 & 139.1 & 119.8 & 60.1 \\
\hline 1900 & 123.3 & 326.0 & 128.7 & 461.0 & 165.1 & 365.4 & 138.4 & 119.2 & 59.9 \\
\hline 1910 & 122.6 & 324.0 & 128.1 & 458.2 & 164.4 & 363.1 & 137.7 & 118.5 & 59.7 \\
\hline 1920 & 121.9 & 322.0 & 127.6 & 455.5 & 163.7 & 360.9 & 137.0 & 117.9 & 59.5 \\
\hline 1930 & 121.3 & 320.1 & 127.1 & 452.9 & 163.1 & 358.6 & 136.4 & 117.3 & 59.3 \\
\hline 1940 & 120.7 & 318.1 & 126.5 & 450.2 & 162.4 & 356.4 & 135.7 & 116.6 & 59.1 \\
\hline 1950 & 120.0 & 316.2 & 126.0 & 447.5 & 161.7 & 354.2 & 135.0 & 116.0 & 58.8 \\
\hline 1960 & 119.4 & 314.3 & 125.5 & 444.9 & 161.1 & 352.0 & 134.4 & 115.4 & 58.6 \\
\hline 1970 & 118.8 & 312.3 & 125.0 & 442.3 & 160.4 & 349.8 & 133.7 & 114.8 & 58.4 \\
\hline 1980 & 118.2 & 310.5 & 124.4 & 439.7 & 159.8 & 347.7 & 133.1 & 114.2 & 58.2 \\
\hline 1990 & 117.5 & 308.6 & 123.9 & 437.1 & 159.2 & 345.5 & 132.4 & 113.6 & 58.0 \\
\hline 2000 & 116.9 & 306.7 & 123.4 & 434.6 & 158.5 & 343.3 & 131.8 & 113.0 & 57.8 \\
\hline 2010 & 116.3 & 304.9 & 122.9 & 432.0 & 157.9 & 341.2 & 131.2 & 112.4 & 57.6 \\
\hline 2020 & 115.7 & 303.0 & 122.4 & 429.5 & 157.3 & 339.1 & 130.6 & 111.8 & 57.5 \\
\hline 2030 & 115.1 & 301.2 & 121.9 & 427.0 & 156.6 & 337.0 & 129.9 & 111.2 & 57.3 \\
\hline 2040 & 114.5 & 299.4 & 121.4 & 424.5 & 156.0 & 334.9 & 129.3 & 110.6 & 57.1 \\
\hline 2050 & 114.0 & 297.6 & 120.9 & 422.0 & 155.4 & 332.8 & 128.7 & 110.1 & 56.9 \\
\hline 2060 & 113.4 & 295.8 & 120.4 & 419.5 & 154.8 & 330.7 & 128.1 & 109.5 & 56.7 \\
\hline 2070 & 112.8 & 294.0 & 119.9 & 417.1 & 154.2 & 328.7 & 127.5 & 108.9 & 56.5 \\
\hline 2080 & 112.2 & 292.3 & 119.4 & 414.7 & 153.6 & 326.6 & 126.9 & 108.4 & 56.3 \\
\hline 2090 & 111.7 & 290.5 & 118.9 & 412.3 & 153.0 & 324.6 & 126.3 & 107.8 & 56.1 \\
\hline 2100 & 111.1 & 288.8 & 118.5 & 409.9 & 152.3 & 322.6 & 125.8 & 107.3 & 56.0 \\
\hline 2110 & 110.5 & 287.0 & 118.0 & 407.5 & 151.7 & 320.6 & 125.2 & 106.7 & 55.8 \\
\hline 2120 & 110.0 & 285.3 & 117.5 & 405.1 & 151.2 & 318.6 & 124.6 & 106.2 & 55.6 \\
\hline 2130 & 109.4 & 283.6 & 117.0 & 402.8 & 150.6 & 316.6 & 124.0 & 105.6 & 55.4 \\
\hline 2140 & 108.9 & 282.0 & 116.5 & 400.4 & 150.0 & 314.7 & 123.5 & 105.1 & 55.2 \\
\hline 2150 & 108.3 & 280.3 & 116.1 & 398.1 & 149.4 & 312.7 & 122.9 & 104.5 & 55.1 \\
\hline 2160 & 107.8 & 278.6 & 115.6 & 395.8 & 148.8 & 310.8 & 122.4 & 104.0 & 54.9 \\
\hline 2170 & 107.3 & 277.0 & 115.1 & 393.5 & 148.2 & 308.8 & 121.8 & 103.5 & 54.7 \\
\hline 2180 & 106.7 & 275.3 & 114.7 & 391.3 & 147.6 & 306.9 & 121.3 & 103.0 & 54.6 \\
\hline 2190 & 106.2 & 273.7 & 114.2 & 389.0 & 147.1 & 305.0 & 120.7 & 102.5 & 54.4 \\
\hline 2200 & 105.7 & 272.1 & 113.8 & 386.8 & 146.5 & 303.2 & 120.2 & 102.0 & 54.2 \\
\hline 2210 & 105.2 & 270.5 & 113.3 & 384.6 & 145.9 & 301.3 & 119.7 & 101.4 & 54.1 \\
\hline 2220 & 104.7 & 268.9 & 112.9 & 382.3 & 145.4 & 299.4 & 119.1 & 100.9 & 53.9 \\
\hline 2230 & 104.2 & 267.4 & 112.4 & 380.2 & 144.8 & 297.6 & 118.6 & 100.4 & 53.7 \\
\hline 2240 & 103.7 & 265.8 & 112.0 & 378.0 & 144.2 & 295.7 & 118.1 & 99.9 & 53.6 \\
\hline 2250 & 103.1 & 264.2 & 111.5 & 375.8 & 143.7 & 293.9 & 117.6 & 99.5 & 53.4 \\
\hline 2260 & 102.7 & 262.7 & 111.1 & 373.7 & 143.1 & 292.1 & 117.1 & 99.0 & 53.2 \\
\hline 2270 & 102.2 & 261.2 & 110.6 & 371.5 & 142.6 & 290.3 & 116.5 & 98.5 & 53.1 \\
\hline 2280 & 101.7 & 259.6 & 110.2 & 369.4 & 142.0 & 288.5 & 116.0 & 98.0 & 52.9 \\
\hline
\end{tabular}




\begin{tabular}{|c|c|c|c|c|c|c|c|c|c|}
\hline $\begin{array}{l}\text { Time } \\
(\min )\end{array}$ & $\begin{array}{c}\text { Bottom } \\
\text { (C) }\end{array}$ & $\begin{array}{c}15 \\
\text { Inch } \\
\text { Center } \\
\text { (C) }\end{array}$ & $\begin{array}{l}15 \text { inch } \\
\text { Surface } \\
\text { (C) }\end{array}$ & $\begin{array}{l}51 \text { inch } \\
\text { Center } \\
\text { (C) }\end{array}$ & $\begin{array}{l}51 \text { inch } \\
\text { Surface } \\
\text { (C) }\end{array}$ & $\begin{array}{l}87 \text { inch } \\
\text { Center } \\
\text { (C) }\end{array}$ & $\begin{array}{l}87 \text { inch } \\
\text { Surface } \\
\text { (C) }\end{array}$ & $\begin{array}{l}99 \text { inch } \\
\text { Center } \\
\text { (C) }\end{array}$ & $\begin{array}{l}99 \text { inch } \\
\text { Surface } \\
\text { (C) }\end{array}$ \\
\hline 2290 & 101.2 & 258.1 & 109.8 & 367.3 & 141.5 & 286.8 & 115.5 & 97.5 & 52.8 \\
\hline 2300 & 100.7 & 256.6 & 109.3 & 365.2 & 140.9 & 285.0 & 115.0 & 97.1 & 52.6 \\
\hline 2310 & 100.2 & 255.2 & 108.9 & 363.1 & 140.4 & 283.3 & 114.5 & 96.6 & 52.5 \\
\hline 2320 & 99.7 & 253.7 & 108.5 & 361.1 & 139.9 & 281.6 & 114.0 & 96.1 & 52.3 \\
\hline 2330 & 99.3 & 252.2 & 108.0 & 359.0 & 139.3 & 279.8 & 113.6 & 95.7 & 52.2 \\
\hline 2340 & 98.8 & 250.8 & 107.6 & 357.0 & 138.8 & 278.1 & 113.1 & 95.2 & 52.0 \\
\hline 2350 & 98.3 & 249.3 & 107.2 & 355.0 & 138.3 & 276.4 & 112.6 & 94.8 & 51.9 \\
\hline 2360 & 97.9 & 247.9 & 106.8 & 353.0 & 137.7 & 274.8 & 112.1 & 94.3 & 51.7 \\
\hline 2370 & 97.4 & 246.5 & 106.3 & 351.0 & 137.2 & 273.1 & 111.6 & 93.9 & 51.6 \\
\hline 2380 & 97.0 & 245.0 & 105.9 & 349.0 & 136.7 & 271.4 & 111.2 & 93.4 & 51.4 \\
\hline 2390 & 96.5 & 243.6 & 105.5 & 347.1 & 136.2 & 269.8 & 110.7 & 93.0 & 51.3 \\
\hline 2400 & 96.1 & 242.3 & 105.1 & 345.1 & 135.7 & 268.2 & 110.2 & 92.5 & 51.1 \\
\hline 2410 & 95.6 & 240.9 & 104.7 & 343.2 & 135.1 & 266.6 & 109.8 & 92.1 & 51.0 \\
\hline 2420 & 95.2 & 239.5 & 104.3 & 341.2 & 134.6 & 265.0 & 109.3 & 91.7 & 50.8 \\
\hline 2430 & 94.7 & 238.1 & 103.9 & 339.3 & 134.1 & 263.4 & 108.8 & 91.2 & 50.7 \\
\hline 2440 & 94.3 & 236.8 & 103.5 & 337.4 & 133.6 & 261.8 & 108.4 & 90.8 & 50.5 \\
\hline 2450 & 93.9 & 235.4 & 103.1 & 335.5 & 133.1 & 260.2 & 107.9 & 90.4 & 50.4 \\
\hline 2460 & 93.5 & 234.1 & 102.7 & 333.7 & 132.6 & 258.7 & 107.5 & 90.0 & 50.3 \\
\hline 2470 & 93.0 & 232.8 & 102.3 & 331.8 & 132.1 & 257.1 & 107.0 & 89.6 & 50.1 \\
\hline 2480 & 92.6 & 231.5 & 101.9 & 330.0 & 131.6 & 255.6 & 106.6 & 89.2 & 50.0 \\
\hline 2490 & 92.2 & 230.2 & 101.5 & 328.1 & 131.1 & 254.0 & 106.1 & 88.8 & 49.9 \\
\hline 2500 & 91.8 & 228.9 & 101.1 & 326.3 & 130.6 & 252.5 & 105.7 & 88.3 & 49.7 \\
\hline 2510 & 91.4 & 227.6 & 100.7 & 324.5 & 130.1 & 251.0 & 105.3 & 87.9 & 49.6 \\
\hline 2520 & 91.0 & 226.3 & 100.4 & 322.7 & 129.7 & 249.5 & 104.8 & 87.6 & 49.5 \\
\hline 2530 & 90.5 & 225.1 & 100.0 & 320.9 & 129.2 & 248.1 & 104.4 & 87.2 & 49.3 \\
\hline 2540 & 90.1 & 223.8 & 99.6 & 319.2 & 128.7 & 246.6 & 104.0 & 86.8 & 49.2 \\
\hline 2550 & 89.7 & 222.6 & 99.2 & 317.4 & 128.2 & 245.1 & 103.6 & 86.4 & 49.1 \\
\hline 2560 & 89.3 & 221.3 & 98.8 & 315.7 & 127.7 & 243.7 & 103.1 & 86.0 & 48.9 \\
\hline 2570 & 88.9 & 220.1 & 98.5 & 313.9 & 127.3 & 242.3 & 102.7 & 85.6 & 48.8 \\
\hline 2580 & 88.6 & 218.9 & 98.1 & 312.2 & 126.8 & 240.8 & 102.3 & 85.2 & 48.7 \\
\hline 2590 & 88.2 & 217.7 & 97.7 & 310.5 & 126.3 & 239.4 & 101.9 & 84.8 & 48.6 \\
\hline 2600 & 87.8 & 216.4 & 97.4 & 308.8 & 125.9 & 238.0 & 101.5 & 84.5 & 48.4 \\
\hline 2610 & 87.4 & 215.3 & 97.0 & 307.1 & 125.4 & 236.6 & 101.1 & 84.1 & 48.3 \\
\hline 2620 & 87.0 & 214.1 & 96.6 & 305.4 & 124.9 & 235.3 & 100.7 & 83.7 & 48.2 \\
\hline 2630 & 86.6 & 212.9 & 96.3 & 303.8 & 124.5 & 233.9 & 100.3 & 83.4 & 48.1 \\
\hline 2640 & 86.3 & 211.7 & 95.9 & 302.1 & 124.0 & 232.5 & 99.9 & 83.0 & 47.9 \\
\hline 2650 & 85.9 & 210.6 & 95.5 & 300.5 & 123.5 & 231.2 & 99.5 & 82.6 & 47.8 \\
\hline 2660 & 85.5 & 209.4 & 95.2 & 298.8 & 123.1 & 229.8 & 99.1 & 82.3 & 47.7 \\
\hline 2670 & 85.2 & 208.3 & 94.8 & 297.2 & 122.6 & 228.5 & 98.7 & 81.9 & 47.6 \\
\hline 2680 & 84.8 & 207.1 & 94.5 & 295.6 & 122.2 & 227.2 & 98.3 & 81.6 & 47.5 \\
\hline 2690 & 84.4 & 206.0 & 94.1 & 294.0 & 121.7 & 225.9 & 97.9 & 81.2 & 47.3 \\
\hline 2700 & 84.1 & 204.9 & 93.8 & 292.4 & 121.3 & 224.6 & 97.5 & 80.9 & 47.2 \\
\hline 2710 & 83.7 & 203.8 & 93.4 & 290.8 & 120.9 & 223.3 & 97.1 & 80.5 & 47.1 \\
\hline 2720 & 83.4 & 202.7 & 93.1 & 289.3 & 120.4 & 222.0 & 96.8 & 80.2 & 47.0 \\
\hline 2730 & 83.0 & 201.6 & 92.8 & 287.7 & 120.0 & 220.7 & 96.4 & 79.9 & 46.9 \\
\hline 2740 & 82.7 & 200.5 & 92.4 & 286.2 & 119.5 & 219.5 & 96.0 & 79.5 & 46.8 \\
\hline 2750 & 82.3 & 199.4 & 92.1 & 284.6 & 119.1 & 218.2 & 95.6 & 79.2 & 46.6 \\
\hline
\end{tabular}


COMSOL Multiphysics Model for DWPF Canister Filling

\begin{tabular}{|c|c|c|c|c|c|c|c|c|c|}
\hline $\begin{array}{l}\text { Time } \\
(\min )\end{array}$ & $\begin{array}{l}\text { Bottom } \\
\text { (C) }\end{array}$ & $\begin{array}{c}15 \\
\text { Inch } \\
\text { Center } \\
\text { (C) }\end{array}$ & $\begin{array}{l}15 \text { inch } \\
\text { Surface } \\
\text { (C) }\end{array}$ & $\begin{array}{l}51 \text { inch } \\
\text { Center } \\
\text { (C) }\end{array}$ & $\begin{array}{c}51 \text { inch } \\
\text { Surface } \\
\text { (C) }\end{array}$ & $\begin{array}{l}87 \text { inch } \\
\text { Center } \\
\text { (C) }\end{array}$ & $\begin{array}{c}87 \text { inch } \\
\text { Surface } \\
\text { (C) }\end{array}$ & $\begin{array}{l}99 \text { inch } \\
\text { Center } \\
\text { (C) }\end{array}$ & $\begin{array}{l}99 \text { inch } \\
\text { Surface } \\
\text { (C) }\end{array}$ \\
\hline 2760 & 82.0 & 198.3 & 91.7 & 283.1 & 118.7 & 217.0 & 95.3 & 78.8 & 46.5 \\
\hline 2770 & 81.6 & 197.2 & 91.4 & 281.6 & 118.2 & 215.7 & 94.9 & 78.5 & 46.4 \\
\hline 2780 & 81.3 & 196.2 & 91.1 & 280.1 & 117.8 & 214.5 & 94.5 & 78.2 & 46.3 \\
\hline 2790 & 81.0 & 195.1 & 90.7 & 278.6 & 117.4 & 213.3 & 94.2 & 77.9 & 46.2 \\
\hline 2800 & 80.6 & 194.1 & 90.4 & 277.1 & 117.0 & 212.1 & 93.8 & 77.5 & 46.1 \\
\hline 2810 & 80.3 & 193.1 & 90.1 & 275.6 & 116.5 & 210.9 & 93.4 & 77.2 & 46.0 \\
\hline 2820 & 80.0 & 192.0 & 89.7 & 274.2 & 116.1 & 209.7 & 93.1 & 76.9 & 45.9 \\
\hline 2830 & 79.6 & 191.0 & 89.4 & 272.7 & 115.7 & 208.5 & 92.7 & 76.6 & 45.8 \\
\hline 2840 & 79.3 & 190.0 & 89.1 & 271.3 & 115.3 & 207.4 & 92.4 & 76.3 & 45.7 \\
\hline 2850 & 79.0 & 189.0 & 88.8 & 269.9 & 114.9 & 206.2 & 92.0 & 76.0 & 45.5 \\
\hline 2860 & 78.7 & 188.0 & 88.5 & 268.4 & 114.5 & 205.0 & 91.7 & 75.7 & 45.4 \\
\hline 2870 & 78.3 & 187.0 & 88.1 & 267.0 & 114.1 & 203.9 & 91.3 & 75.4 & 45.3 \\
\hline 2880 & 78.0 & 186.0 & 87.8 & 265.6 & 113.6 & 202.8 & 91.0 & 75.0 & 45.2 \\
\hline 2890 & 77.7 & 185.0 & 87.5 & 264.2 & 113.2 & 201.6 & 90.6 & 74.7 & 45.1 \\
\hline 2900 & 77.4 & 184.1 & 87.2 & 262.8 & 112.8 & 200.5 & 90.3 & 74.4 & 45.0 \\
\hline 2910 & 77.1 & 183.1 & 86.9 & 261.4 & 112.4 & 199.4 & 89.9 & 74.2 & 44.9 \\
\hline 2920 & 76.8 & 182.1 & 86.6 & 260.1 & 112.0 & 198.3 & 89.6 & 73.9 & 44.8 \\
\hline 2930 & 76.5 & 181.2 & 86.3 & 258.7 & 111.6 & 197.2 & 89.3 & 73.6 & 44.7 \\
\hline 2940 & 76.2 & 180.2 & 86.0 & 257.4 & 111.2 & 196.1 & 88.9 & 73.3 & 44.6 \\
\hline 2950 & 75.9 & 179.3 & 85.7 & 256.0 & 110.8 & 195.0 & 88.6 & 73.0 & 44.5 \\
\hline 2960 & 75.6 & 178.4 & 85.4 & 254.7 & 110.5 & 194.0 & 88.3 & 72.7 & 44.4 \\
\hline 2970 & 75.3 & 177.4 & 85.1 & 253.4 & 110.1 & 192.9 & 87.9 & 72.4 & 44.3 \\
\hline 2980 & 75.0 & 176.5 & 84.8 & 252.1 & 109.7 & 191.8 & 87.6 & 72.1 & 44.2 \\
\hline 2990 & 74.7 & 175.6 & 84.5 & 250.8 & 109.3 & 190.8 & 87.3 & 71.8 & 44.1 \\
\hline 3000 & 74.4 & 174.7 & 84.2 & 249.5 & 108.9 & 189.8 & 87.0 & 71.6 & 44.0 \\
\hline
\end{tabular}




\section{Distribution}

J. W. Amoroso, 999-W

A. B. Barnes, 999-W

A. L. Billings, 999-W

C. L. Crawford, 773-42A

D. A. Crowley, 773-43A

A. P. Fellinger, 773-41A

S. D. Fink, 773-A

K. M. Fox, 999-W

B. J. Giddings, 786-5A

N.K. Gupta, 703-41A

C. C. Herman, 999-W

C. M. Jantzen, 773-A

F. C. Johnson, 999-W

M.R. Kesterson, 703-41A

P.L. Lee, 703-41A

S. L. Marra, 773-A

D. K. Peeler, 999-W

F. M. Pennebaker, 773-42A

M. E. Stone, 999-W

D.A. Tamburello, 703-41A

W. R. Wilmarth, 773-A 